\title{
Hybrid Plasmonics for Energy Harvesting and Sensing of Radiation and Heat
}

\section{Mina Shiran Chaharsoughi}




\title{
Hybrid Plasmonics for Energy Harvesting and Sensing of Radiation and Heat
}

\author{
Mina Shiran Chaharsoughi
}

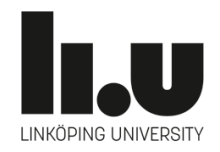

Department of Science and Technology, Laboratory of Organic Electronics

Linköpings universitet, SE-60221 Norrköping, Sweden

Norrköping 2020 
During the course of the research underlying this thesis, Mina Shiran Chaharsoughi was enrolled in Agora Materiae, a multidisciplinary doctoral programme at Linköping University, Sweden.

Shiran Chaharsoughi, Mina

Hybrid Plasmonics for Energy Harvesting and Sensing of Radiation and Heat Linköping Studies in Science and Technology. Dissertations. No. 2045

(c) Copyright 2020 Mina Shiran Chaharsoughi, unless otherwise noted Cover by Mina Shiran Chaharsoughi and Johannes Gladisch, an illustration of plasmonic heating in an array of gold nanodisks illuminated by a beam of light. Printed in Sweden by LiU-Tryck, Linköping 2020

ISSN: 0345-7524

ISBN: 978-91-7929-906-4

Electronic publication: http://www.ep.liu.se 
"The man of science has learned to believe in justification, not by faith, but by verification."

Thomas H. Huxley 


\section{Abstract:}

The special optical properties of subwavelength metallic structures have opened up for numerous applications in different fields. The interaction of light with metal nanostructures leads to the excitation of collective oscillations of conduction-band electrons, known as plasmons. These plasmon excitations are responsible for the high absorption and high scattering of light in metallic nanostructures. High absorption of light and the subsequent temperature increase in the nanostructures make them suitable as point-like heat sources that can be controlled remotely by light.

The research presented in this thesis focuses on the development and studies of hybrid devices that combine light-induced heating in plasmonic nanostructures with other materials and systems. Particular focus is put on hybrid organic-inorganic systems for applications in energy harvesting as well as in heat and radiation sensing. Harvesting energy from light fluctuations was achieved in a hybrid device consisting of plasmonic gold nanodisk arrays and a pyroelectric copolymer. In this concept, fast and efficient light-induced heating in the gold nanodisks modulated the temperature of the pyroelectric layer, which could be used to extract electrical energy from fluctuations in simulated sunlight.

Integrating plasmonic nanostructures with complementary materials can also provide novel hybrid sensors, for monitoring of temperature, heat flux and radiation. In this thesis work, a hybrid sensor was designed based on the combination of a plasmonic gold nanohole layer with a pyroelectric copolymer and an ionic thermoelectric gel. The gold nanohole arrays acted both as broadband light absorbers in the visible to near-infrared spectral range of the solar spectrum and also as one of the electrodes of the sensor. In contrast to the constituent components when used separately, the hybrid sensor could provide both fast and stable signals upon heat or radiation stimuli, as well as enhanced equilibrium signals.

Furthermore, a concept for heat and radiation mapping was developed that was highly sensitive and stable despite its simple structure. The concept consisted of a gellike electrolyte connecting two separated metal nanohole electrodes on a substrate. Resembling traditional thermocouples, this concept could autonomously detect temperature changes but with several orders of magnitudes higher sensitivity. Owing to its promising sensing properties as well as its compatibility with inexpensive mass production methods on flexible substrates, such concept may be particularly interesting for electronic skin applications for health monitoring and for humanoid robotics. Finally, we improved the possibilities for the temperature mapping of the concept by modifying the structure from lateral to vertical form. Similar to the lateral 
device, the vertical temperature sensor showed high temperature sensitivity and stability in producing signals upon temperature changes. 


\section{Populärvetenskaplig sammanfattning:}

Nanostrukturer av metall, vars dimensioner är cirka 1000 gånger mindre än vidden på ett hårstrå, har egendomliga optiska egenskaperna som möjliggjort många olika typer av applikationer. De optiska egenskaperna bygger på att interaktionen mellan ljus och metallnanostrukturer ger upphov till kollektiva oscillationer av elektronerna i metallen, så kallade plasmoner. Dessa plasmon-excitationer leder bland annat till hög absorption och spridningen av ljus i metalliska nanostrukturer. Hög absorption av ljus leder i sin tur till värmeutveckling i nanostrukturerna, vilket gör dem till effektiva värmekällor som kan kontrolleras på avstånd med ljus.

I forskningen som presenteras i den här avhandlingen presenteras hybrida system som kombinerar värmeutveckling i metallnanostrukturer med andra material och koncept. Vi har framför allt utvecklat och studerat hybrida system som kan användas för energiutvinning eller som sensorer för ljus och värme. I en första studie visar vi att energi kan utvinnas från fluktuationer i ljusintensitet, genom en hybridkomponent som kombinerar plasmoniska guldnanodiskar med en så-kallad pyroelektrisk polymer. Ljusinducerad uppvärmning av nanodiskarna generatorrade temperaturen $\mathrm{i}$ det pyroelektriska lagret, vilket kunde generera energi från fluktuationer i simulerat solljus under ett fladdrande löv.

Genom att integrera plasmoniska nanostrukturer med komplementära material var det även möjligt att skapa nya sensorer för att monitorera temperatur och ljusexponering. Vår hybridsensor kombinerar guldnanohål med en pyroelektrisk polymer samt en jonisk termoelektrisk gel. Guldnanohålen användes både för att absorbera ljus och som en av elektroderna i sensorn. Hybridsensorn möjliggjorde både snabb och stabil avläsning av ljus och värme, som inte är möjligt om de aktiva materialen används var för sig.

Vidare så utvecklades ett koncept för detektion av värme och ljus med hög känslighet trots en mycket enkel struktur. Strukturen består av två elektroder med nanohål som är sammanbundna med en gel-elektrolyt. Den är därmed lik ett klassiskt termoelement, men har en känslighet som är flera storleksordningar högre. Dessa sensorer skulle kunna användas till applikationer så som elektronisk hud för hälsoövervakning eller inom robotik, och de skulle kunna tillverkas till låg kostnad på flexibla substrat. Slutligen så lyckades vi förbättra förutsättningarna för temperaturmappningen hos dessa temperatursensorer genom att bygga dem i en vertikal struktur. Likt den laterala sensorn så hade även den vertikala temperatursensorn hög känslighet och bra stabilitet. 


\section{List of publications}

Papers included in this thesis

\section{Paper I}

Hybrid Plasmonic and Pyroelectric Harvesting of Light Fluctuations

Mina Shiran Chaharsoughi, Daniel Tordera, Andrea Grimoldi, Isak Engquist, Magnus Berggren, Simone Fabiano, Magnus P. Jonsson

Advanced Optical Materials 2018, 6 (11), 1701051. doi:10.1002/adom.201701051

Contribution: I performed all the processes related to the device fabrication and characterization and data analysis. I wrote the first draft and contributed to the final editing of the manuscript.

\section{Paper II}

\section{Thermodiffusion-Assisted Pyroelectrics-Enabling Rapid and Stable Heat and Radiation Sensing} Mina Shiran Chaharsoughi, Dan Zhao, Xavier Crispin, Simone Fabiano, and Magnus P. Jonsson Advanced Functional Materials 2019, 29 (28), 1900572. doi:10.1002/adfm.201900572

Contribution: I carried out the experimental work related to the device fabrication and characterization, some together with D.Z. I contributed to analyze and interpret the data and wrote a large part of the first draft and edited the final version of the manuscript.

\section{Paper III}

\section{Ultrasensitive Electrolyte-Assisted Temperature Sensor}

Mina Shiran Chaharsoughi, Jesper Edberg, Peter Andersson Ersman, Xavier Crispin, Dan Zhao, and Magnus P. Jonsson Submitted for publication

Contribution: I conducted the experimental work related to device fabrication and optical measurements. I took part in the discussion of the results and contributed to writing the first draft and editing the final version of the manuscript. 


\section{Paper IV}

Vertical Hybrid Metasurface Supercapacitors for Heat and Radiation Sensing Mina Shiran Chaharsoughi, Ayesha Sultana, Xavier Crispin, Dan Zhao, and Magnus P. Jonsson.

In manuscript

Contribution: I performed the device fabrication and took part in measurements and data analysis. I wrote the majority of the manuscript.

\section{Papers not included in this thesis}

\section{Hybrid Plasmonic Metasurfaces}

Evan S. H. Kang, Mina Shiran Chaharsoughi, Stefano Rossi and Magnus P. Jonsson Journal of Applied Physics 2019, 126, 140901 (Perspective). doi:10.1063/1.5116885

Contribution: I contributed to writing the first draft and editing the final version of the manuscript.

\section{Conductive Polymer Nanoantennas for Dynamic Organic Plasmonics}

Shangzhi Chen, Evan S. H. Kang, Mina Shiran Chaharsoughi, Vallery Stanishev, Philipp Kühne, Hengda Sun, Chuanfei Wang, Mats Fahlman, Simone Fabiano, Vanya Darakchieva, and Magnus P. Jonsson

Nature Nanotechnology 2019. doi:10.1038/s41565-019-0583-y

Contribution: I contributed to the experimental work and editing the final version of the manuscript. 


\section{Acronyms}

\begin{tabular}{|c|c|}
\hline CL & Colloidal Lithography \\
\hline EBL & Electron Beam Lithography \\
\hline EDLC & Electric Double-Layer Capacitor \\
\hline EMIM & 1-ethyl-3-methylimidazolium \\
\hline [EMIM] $\left[\mathrm{ESO}_{4}\right]$ & 1-ethyl-3-methylimidazolium ethylsulfate \\
\hline e-skin & electronic skin \\
\hline FDTD & Finite-Difference Time-Domain \\
\hline FEM & Finite Element Method \\
\hline FIB & Focused-ion Beam Lithography \\
\hline GDM & Green’s Dyadic Method \\
\hline HCL & Hole-mask Colloidal Lithography \\
\hline IL & Ionic Liquid \\
\hline ITO & Indium Tin Oxide \\
\hline LSPR & Localized Surface Plasmon Resonance \\
\hline PNIPAM & Poly(N-isopropylacrylamide) \\
\hline PMMA & Poly(methyl methacrylate) \\
\hline PVC & Poly(vinyl chloride) \\
\hline PVDF & Poly(vinylidene fluoride) \\
\hline PVDF-HFP & Poly(vinylidene fluoride-co-hexafluoropropylene) \\
\hline $\mathrm{P}(\mathrm{VDF}-\mathrm{TrFE})$ & Poly(vinylidenefluoride-co-trifluoroethylene) \\
\hline PZT & Lead Zirconate Titanate \\
\hline RTD & Resistance Temperature Detector \\
\hline SCL & Sparse Colloidal Lithography \\
\hline SEM & Scanning Electron Microscopy \\
\hline SPP & Surface Plasmon Polariton \\
\hline TC & Thermocouple \\
\hline TEG & Thermoelectric Generator \\
\hline TEM & Transmission Electron Microscopy \\
\hline TFSI & Bis(trifluoro-methylsulfonyl)imide \\
\hline
\end{tabular}




\section{Table of Content}

Part I

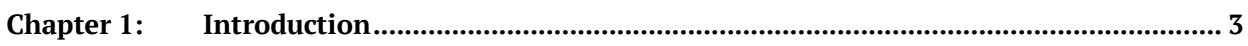

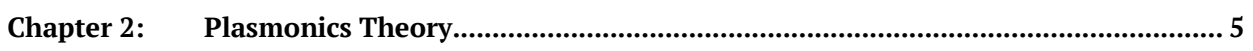

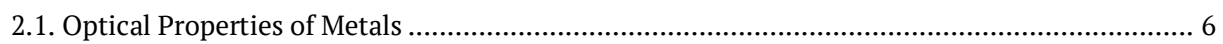

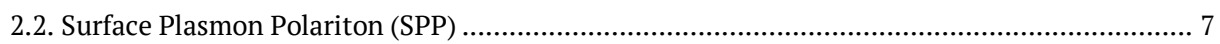

2.3. Localized Surface Plasmon Resonance (LSPR).................................................................... 9

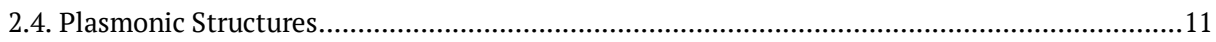

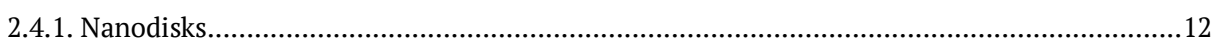

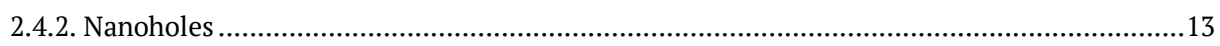

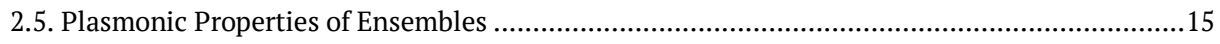

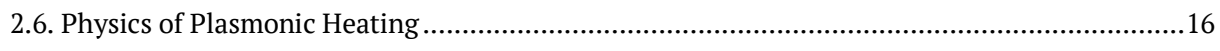

2.6.1. Plasmonic Heating in Ensembles of Gold Nanodisks or Gold Nanoholes...............................18

Chapter 3: Thermal Energy Harvesting and Sensing ........................................................... 21

3.1. Heat-Induced Electric Potential Generation in Materials .....................................................22

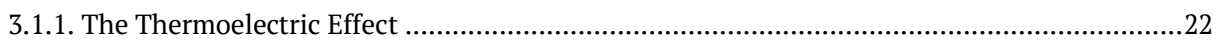

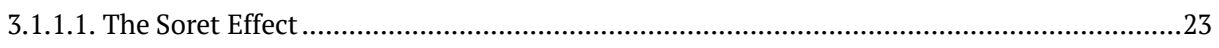

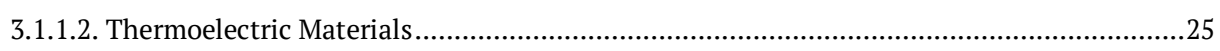

3.1.1.2.1. Ionic Liquids and Their Derivatives for Use in Thermoelectric Applications ...................26

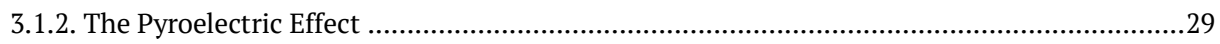

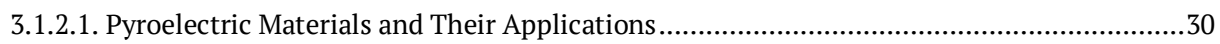

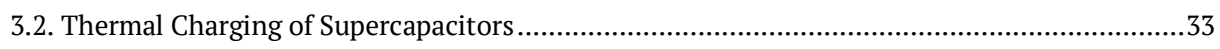

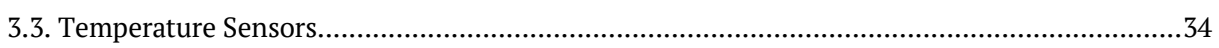

Chapter 4: Methods and Characterization......................................................................... 37

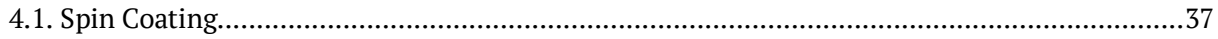

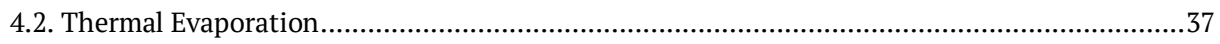

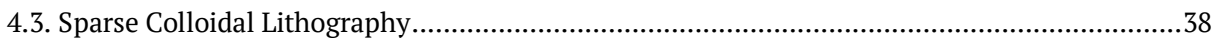

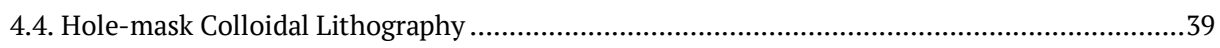

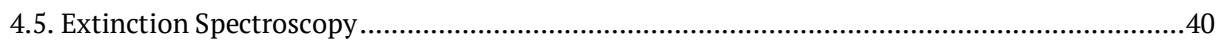

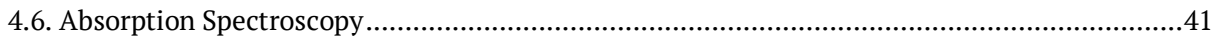

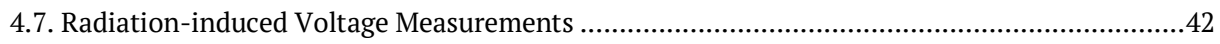

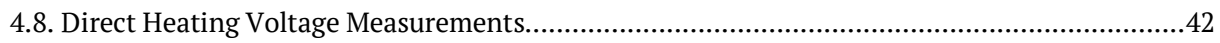

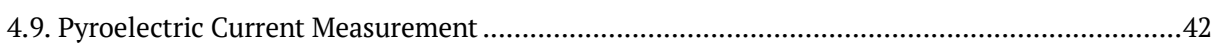

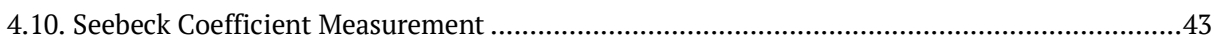




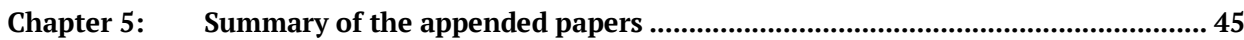

5.1. Harvesting Energy from Light Fluctuations - Paper I.................................................................45

5.2. Three Effects in One Concept for Sensing Radiation and Heat - Paper II .................................48

5.3. Simple Concepts with Outstanding Temperature Sensing Properties - Papers III and IV ........49

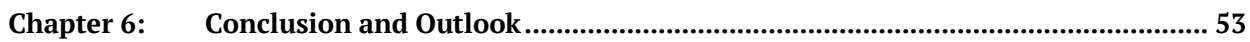

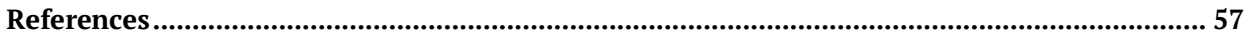

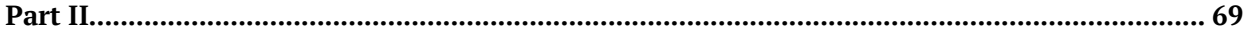




\section{Acknowledgement}

A wise proverb says "it takes a village to raise a child". In the case of this thesis, the child is a metaphor for the knowledge, experience and insight, and the village is all the people who helped me during the last four years to complete this work. I would like to express my gratitude and appreciation to:

Magnus Jonsson, my main supervisor, for giving me this opportunity to work on this interesting project, and for supporting me and shedding light on my way to achieve my goals.

Xavier Crispin and Simone Fabiano, my co-supervisors, for their valuable guidance and inspiring discussions.

Lars Gustavsson, Anna Malmström, Meysam Karami Rad, and Thomas Karlsson for their restless efforts in maintaining the lab at its best condition and for helping me to optimize my performance in the experiments.

Lesley G Bornhöft and Kattis Nordlund for always being so kind to me and help me to handle administrative issues.

Dan and Jesper for rewarding collaboration and support in the lab and fruitful discussions.

Shangzhi, Sampath, Ravi, Samim, and Stefano, my colleagues and friends in Organic Photonic and Nano-Optics group, for being such amazing and supportive labmates. Daniel Tordera, my mentor, for guiding me and being inspiring and cheerful. Evan for interesting discussion especially during taking SEM images.

Johannes, Dan, Evan, Nadia, Fareed, Miriam, and Sämi for your valuable comments on the first draft of this thesis.

All former and current members of the Laboratory of Organic Electronics that provided a pleasant and cheerful environment at work. Zia Ullah Khan and Ujwala for helping me out in the lab and being open to answer all my questions. Dagmavi and Fareed for cheering me up when I was down and were always there for me when I needed help. Donata, Ellen, Felipe, Maria and Elina for making me feel welcome when I joined the group. Canyan and Shaobo for their warm friendship and being always eager to help and support me both in the lab and outside work. 
Negar and Arash for being supportive and encouraging friends and helping me to establish my life when I came to Sweden.

Andrea and Eva for all the fun moments in the climbing gym where I could free my mind. Harpal and Chiara for sharing those delicious and pleasant moments in Burger and Bangers after the climbing gym. Nadia for inspiring me and lending me an ear to my concerns and suggesting perfect remedies. Roudabeh and Najmeh for making ordinary moments extraordinary and all the laughter. Farzaneh and Sämi for being nice friends and amazing office neighbors sharing their cookies and jokes with me. Mahshid, Niki, and Saghi for your kindness, well-wishes, and being there for me whenever I needed a friend.

A special word of thank to my mother and my brother, for encouraging me to achieve my best and filling my heart with their love to overcome the hardships of living distant from them. Undoubtedly, the love and support I received from my father, is a major source of motivation for me to achieve personal and professional goals. At my early age, he instilled in me a strong passion for learning, which ultimately led me to be in Sweden and studying my PhD. I owe him a lot and I dedicate this thesis to him and my mother.

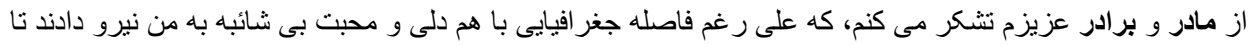

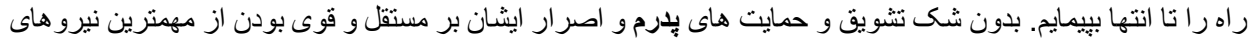

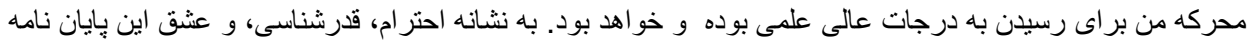
را به مادر و بدرم تقديم ميكنم.

In particular thanks to Johannes (JoJo) who supported me with his unconditional love. Thank you for always having faith in me and helping me to overcome stressful moments. 


\section{Part I}

\section{Background}




\section{Chapter 1: Introduction}

Metal nanostructures have so-called plasmonic properties, which make them attractive tools for manipulation of light at the nanoscale. The interaction of light with these structures leads to a large optical field enhancement, high scattering and high absorption of light. ${ }^{[1-5]}$ Such interesting optical properties of metal nanostructures originate from resonant oscillation of their conduction-band electrons upon light illumination, known as localized surface plasmon resonance (LSPR). $\left.{ }^{[3,} 6\right]$ LSPRs and corresponding high absorption of light allow metallic nanostructures to efficiently convert light to thermal energy at excitation wavelengths which are tunable via manipulating different parameters, such as the shape of the nanostructure. ${ }^{[7]}$ The plasmonic photothermal effect enables efficient and selective control of heat generation at the nanoscale, which could be useful in many applications in various fields. ${ }^{[1,8,9]}$ In biomedicine, the effect may enable photothermal therapy of cancerous cells and curing disease via delivering drugs to specific parts of the human body. ${ }^{[3,10]}$ In nano-chemistry, plasmonic heating can facilitate or enhance chemical reactions at the nanoscale, by varying the local temperature at the reaction sites. ${ }^{[11]}$ In optofluidics, manipulation of fluid motion at the nanoscale is feasible through photothermal-induced convection by a metallic nanostructure. ${ }^{[12]}$

Integration of plasmonic nanostructures with other materials into hybrid systems broadens the scope of plasmonic heating applications. ${ }^{[4,13,14]}$ The focus of this thesis work is to exploit plasmonic heating in hybrid systems that combine metallic nanostructures with organic materials that have special functionalities, such as pyroelectricity and thermoelectricity. Such hybrid systems provide promising applications ranging from energy harvesting from light fluctuations to sensing radiation and heat.

This thesis is divided into two parts. The first part contains 5 chapters, starting with this introduction. Chapter 2 describes the basics of plasmonics and its two main 
components: surface plasmon polaritons and localized surface plasmons. Furthermore, this chapter introduces different plasmonic structures employed in this thesis and discusses their plasmonic and photothermal properties. Chapter 3 introduces various physical phenomena and the related materials for converting heat to electrical energy and signals. Chapter 4 describes the methods utilized in this thesis, including fabrication of plasmonic structures and hybrid devices. It also outlines the characterization and measurement methods utilized in this thesis. Chapter 5 and 6 discuss main results of this work and future extensions. The second part of this thesis presents the result of the scientific work as four appended papers. Paper I presents a hybrid concept that takes advantage of plasmonic heating in combination with the pyroelectric effect to harvest energy from light fluctuations. Paper II deals with integrating plasmonic heating, pyroelectric effect and thermoelectric effect into a hybrid device to enable a fast and stable temperature and radiation sensing. Paper III and IV present concepts that, despite of their simple structures, can detect variations in temperature via strong self-generated signals, upon both radiation and direct contact with heat stimuli. 


\section{Chapter 2: Plasmonics Theory}

The science of plasmonics studies the interaction of electromagnetic fields with the collective oscillation of free electrons of metallic interfaces or in metallic nanostructures, with one major aim of confining enhanced optical fields at dimensions beyond the diffraction limit. ${ }^{[15-17]}$ Depending on the geometry of metallic nanostructured surfaces, collective oscillation of free electron gas called plasmons can couple to light through surface plasmon polaritons (SPPs) (Chapter 2.2) and localized surface plasmon resonance (LSPRs) (Chapter 2.3). ${ }^{[18-21]}$ While the former can be excited at the interface of a metal and a dielectric medium, resulting in the confinement of the optical fields to the interface, the latter can occur in metallic nanostructures such as nano-spheres or nano-voids with the dimensions smaller than the wavelength of light. ${ }^{[19,20,22-25]}$ Both SPPs and LSPRs in metallic structures are sensitive to small changes of the refractive index of the surroundings, which makes them a powerful tool for nanosensing. ${ }^{[6,24,26]}$

a)

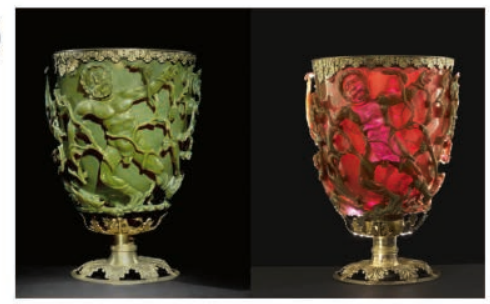

b)

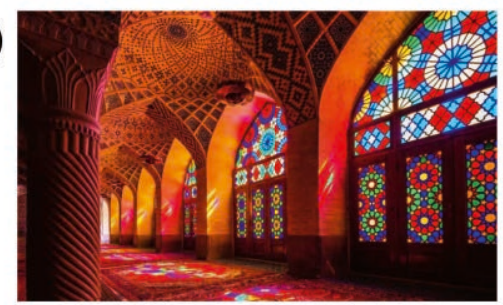

Figure 2-1. a) The Lycurgus cup, 4th-century Roman glass cage cup, appears jade green when lit from the front and red when from backwards (from the British Museum free image service). b) Stained glass in Nasir al-Mulk Mosque built in 1888 in Shiraz, Iran (from reference [27]). 
The LSPR in subwavelength metallic nanostructures is the reason for their unique absorption and scattering optical properties. ${ }^{[28]}$ One of the first attempts to utilize metallic nanoparticles dates back to several hundred years ago when the manufactures used them as means of coloration and decoration of glass objects. The Lycurgus cup (Figure 2-1a) and colorful stained glass in churches and mosques (Figure $2-1 b)$ are examples of those first attempts that have lasted till present day. ${ }^{[16,17,29]}$

Until a couple of decades ago, the application of metallic nanoparticles was mainly defined based on their scattering properties. In most applications, absorption of light by these structures was considered a drawback since it caused photothermal effects and made systems less efficient. Only recently, plasmonic heating (section 2.6) in metallic nanoparticles has been explored in various areas such as chemistry, physics and biology. ${ }^{[4,10,30,31]}$

\subsection{Optical Properties of Metals}

To a first approximation, the optical properties of metals can be explained by treating their conduction electrons as free electrons. The behavior of such electrons is described by the Drude model similar to classical gas molecules, where a gas of free electrons moves against a fixed background of positive ion cores. The interaction of light with such electrons can be explained by permittivity or dielectric function $\varepsilon(\omega)$ as below: ${ }^{[32]}$

$$
\varepsilon(\omega)=1-\frac{\omega_{p}^{2}}{\omega^{2}+i \gamma \omega} \quad(2-1)
$$

where $\omega$ is the angular frequency, $\omega_{p}$ is the plasma frequency $\left(\omega_{p} \approx 1.4 \times 10^{16} \mathrm{~Hz}\right.$ for gold), and $\gamma$ is the collision frequency (in the order of $10^{14} \mathrm{~Hz}$ at room temperature for gold), which indicates the damping of the oscillation of the electron gas due to collision. ${ }^{[32]}$ For metals such as gold and silver with plasma frequencies in the range of $10^{15}-10^{16} \mathrm{~Hz}$ and significantly lower $\gamma$, electrons oscillate faster than the collision frequency so the effect of collisions can often be neglected and equation (2-1) can be rewritten as: ${ }^{[20,32]}$

$$
\varepsilon(\omega)=1-\frac{\omega_{p}^{2}}{\omega^{2}} . \quad(2-2)
$$

For optical frequencies below the plasma frequency, the permittivity gains negative values and the refractive index $(\tilde{n}(\omega))$ becomes purely imaginary $\left(\tilde{n}(\omega)=\varepsilon^{1 / 2}\right)$. In this spectral region, the electric field can penetrate the metal to a certain distance, but not propagate further as an oscillating wave. If the metal is thick enough, the whole incoming wave will be reflected and the metal shows its familiar shiny and colorless 
optical properties. According to the Drude model, at frequencies higher than the plasma frequency, the dielectric function gains positive values approaching 1 and the refractive index becomes real, making the material more transparent in this spectral range. For noble metals such as gold and silver, a full $d$ electronic band lying a few electron volts below the Fermi level creates a highly polarized environment that adds dielectric constant $\varepsilon_{\infty}$ (typically with values $1 \leq \varepsilon_{\infty} \leq 10$ ) to the dielectric function, which modifies equation (2-1) to: ${ }^{[32,33]}$

$$
\varepsilon(\omega)=\varepsilon_{\infty}-\frac{\omega_{p}^{2}}{\omega^{2}+i \gamma \omega} . \quad(2-3)
$$

The validity of the Drude model for aforementioned metals is also limited due to the interband transitions of electrons. Adding more terms like Lorentz-oscillator terms to the dielectric function can improve the validity of this model. ${ }^{[32]}$

\subsection{Surface Plasmon Polariton (SPP)}

In the field of surface science, surface plasmon polaritons (SPPs) are well-known following the pioneering work of Ritchie in 1950s. ${ }^{[20]}$ SPPs are electromagnetic surface waves created by the interaction of light waves with the conductor's free electron gas on the conductor/dielectric interfaces. In this interaction, the electron gas responds collectively by oscillating in resonance with an incoming electromagnetic wave. The SPP waves propagating at the interface between a conductor and a dielectric (Figure $2-2)$ are transverse magnetic waves with two electric field components: one $\left(E_{x}\right)$ parallel to the propagation direction and the other $\left(E_{z}\right)$ normal to the plane of the interface.

As shown in Figure 2-2, $E_{z}$ is highest near the surface and decays exponentially with distance away from the interface, both into the metal and the dielectric medium. The reciprocal value of the component of the wave vector perpendicular to the interface $\left(k_{z}\right)$ of the two media, $\hat{z}=1 /\left|k_{z}\right|$, defines the exponential decay length which is typically a few hundreds of nanometers. ${ }^{[20]}$ The energy of the SPP waves decays while propagating along the interface mainly because of the absorption by the metal. The propagation length $\left(L=\left(2 \operatorname{Im}\left[k_{x}\right]\right)^{-1}\right)$ is defined as the distance at which the intensity of the SPP wave decays by a factor of $1 / \mathrm{e}$, and for metals with low loss such as silver in the visible spectrum is typically between 10 to 100 micrometers. ${ }^{32]}$ 


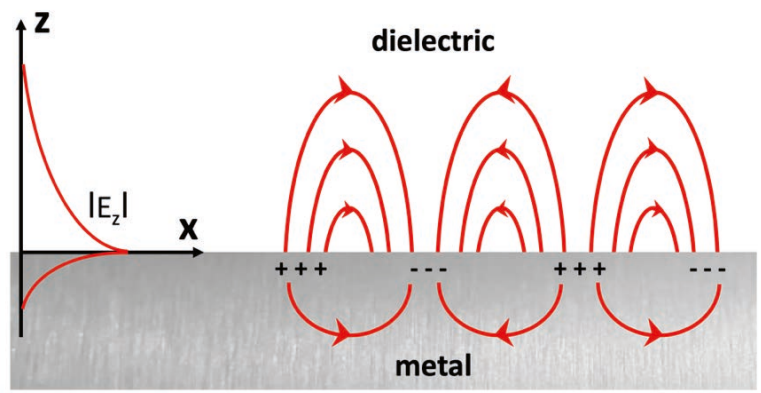

Figure 2-2. Schematic representation of SPP waves propagating at the interface between a dielectric and a metal. The electric field component is enhanced near the surface and decays exponentially with distance away from the interface both into the metal and the dielectric medium. ${ }^{[20]}$

Both the decay length and the propagation length have a strong dependency on frequency and the dielectric function of both the conductor $(\varepsilon)$ and the surrounding dielectric material $\left(\varepsilon_{d}\right)$. In addition, the propagation length depends on the metal/dielectric configuration. ${ }^{[32,34]}$

The dispersion relation for SPPs on a metal dielectric interface gives us the relation between the wave vector $\left(k_{S P P}\right)$ and energy of the SPP: ${ }^{20,34]}$

$$
k_{s p p}=\frac{\omega}{c} \sqrt{\frac{\varepsilon_{d} \varepsilon}{\varepsilon_{d}+\varepsilon}} \quad(2-4)
$$

where $c$ is the speed of light in free space. Figure 2-3 shows the dispersion relation for SPPs on a metal air interface (black line) and the dispersion line of photons in free space (red line, $\omega=c k$, where $k$ is the wave vector). The wave vector of the SPP wave is always higher than that of light for any frequency (Figure 2-3). Hence, a light beam that hits the metal surface from air cannot excite SPPs at the metal interface unless the photon momentum is somehow increased to match the SPP momentum. There are two major optical approaches to overcome this momentum mismatch, namely using a prism coupler or diffraction gratings. ${ }^{[23]}$ For the prism coupling method, such as the Kretschmann configuration and the Otto configuration, the momentum of the photon is enhanced by passing through a high refractive index medium at an angle larger than the critical angle. ${ }^{[23,34,35]}$ The grating contributes with additional momentum within the plane and makes it possible to match both momentum and energy of SPPs, even at normal incidence. ${ }^{[35,36]}$ 


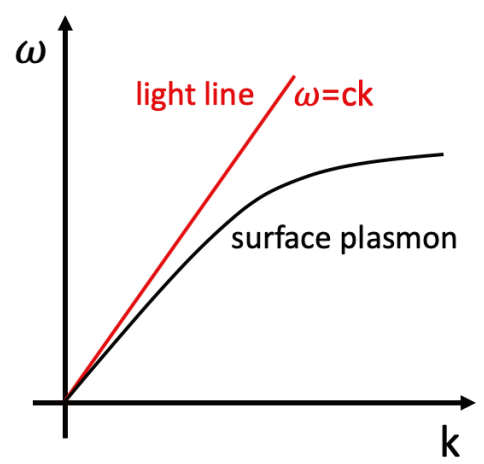

Figure 2-3. Dispersion relation of SPPs on a metal-air interface (black line) and dispersion line of a photon in free space (red line) ${ }^{[20]}$

The possibility to use SPPs to confine optical fields to the interface between metal and dielectric layers has brought many applications for SPPs in photonics, biology and materials science. $\left.{ }^{[4,} 20,33,36\right]$ For instance, SPP waves have been widely used in biosensing applications since they are very sensitive to changes in the dielectric constant of the environment. ${ }^{[4,20]}$ Moreover, the waveguides that support SPP waves have many applications in nanocircuits and nanophotonic devices. ${ }^{[33,36]}$ In-depth information about the properties and applications of SPP waves can be found in these good reviews. ${ }^{[35,37]}$

\subsection{Localized Surface Plasmon Resonance (LSPR)}

Another type of surface plasmonic mode is localized surface plasmon resonance, which is a non-propagating mode supported by closed surfaces, such as metallic nanoparticles smaller than the wavelength of light. ${ }^{[16,38,39]}$ Under illumination, the time-varying electric field of light induces a force on the electron plasma of the nanoparticle and displaces it from its equilibrium position, leading to an uncompensated charge at the surface of the nanoparticle (Figure 2-4) and an induced dipole moment in the nanoparticle. ${ }^{[38]}$ In the quasi-static approximation, where the diameter $(D)$ of the nanoparticle is significantly smaller than the wavelength of incident light $(D<<\lambda)$, the field of incident light can be seen as uniform over the whole nanoparticle volume at any given time. In this approximation, the polarizability $(\alpha(\omega))$ of a sphere with dielectric function of $\varepsilon(\omega)$ surrounded by a medium with dielectric function of $\varepsilon_{d}$ can be described by:[32]

$$
\alpha(\omega)=4 \pi\left(\frac{D}{2}\right)^{3} \frac{\varepsilon(\omega)-\varepsilon_{d}}{\varepsilon(\omega)+2 \varepsilon_{d}} . \quad(2-5)
$$




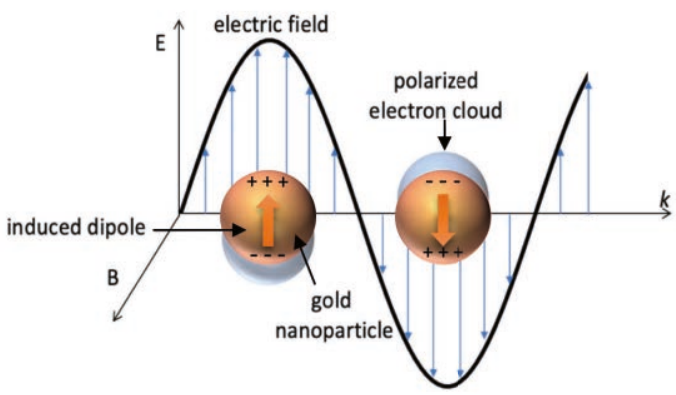

Figure 2-4. The time-varying electric field of light applies a force on the electron plasma of the nanoparticle and induces a dipole moment in the nanoparticle.

In Fröhlich condition, where $\operatorname{Im}[\varepsilon(\omega)]$ is small, the polarizability reaches a maximum when $\operatorname{Re}\left[\varepsilon(\omega)=-2 \varepsilon_{d}\right]$, which gives the resonance frequency $\omega_{M}=\omega_{p} / \sqrt{3}$ for a Drude material. ${ }^{[32]}$ The collective oscillation of the electrons resonates with the incident light, which forms the localized surface plasmon resonance or surface plasmon resonance for short. ${ }^{[32,}{ }^{39]}$ The LSPR frequency is very sensitive to the dielectric medium such that increasing the dielectric constant of the surrounding medium leads to a redshift to the LSPR. This phenomenon enables the application of LSPR for optical sensing, which is especially useful because the phase matching between the momentum of the incident light and the plasmon resonance is not needed and LSPR can be activated by illumination with an ordinary light beam. ${ }^{[3,6,16}$, $17,39,40]$

Illuminating a nanoparticle at the LSPR causes a resonance enhancement in scattering and absorption efficiency of the nanoparticle, which has resulted in many applications in various areas, such as staining and coloration, ${ }^{[41,42]}$ information storage, ${ }^{[43]}$ medical therapy, ${ }^{[44]}$ optofluidics, ${ }^{[45]}$ and bio-sensing. ${ }^{[39]}$ The scattering $\left(\sigma_{\text {scat }}\right)$, absorption $\left(\sigma_{a b s}\right)$ and extinction $\left(\sigma_{\text {ext }}\right)$ cross-sections of a nanoparticle can be expressed by:[31,46]

$$
\begin{gathered}
\sigma_{\text {scat }}=\frac{k^{4}}{6 \pi}|\alpha|^{2}, \quad(2-6) \\
\sigma_{a b s}=k \operatorname{Im}(\alpha)-\frac{k^{4}}{6 \pi}|\alpha|^{2}, \quad(2-7) \\
\sigma_{\text {ext }}=\sigma_{\text {scat }}+\sigma_{a b s}=k \operatorname{Im}(\alpha), \quad(2-8)
\end{gathered}
$$

where $k$ is the wave vector of the incident light. The interaction of light with particles smaller than the wavelength of incident light $(D<<\lambda)$ predominantly leads to excitation of dipole modes. ${ }^{[32,40]}$ However, for particles with sizes comparable to the wavelength of the incident light wavelength, higher order modes such as quadrupole oscillations can also contribute to the extinction cross-section. For such large 
particles, the quasi-static approximation is no longer valid since the particle does not experience a uniform electromagnetic field over its volume. This causes a rapid loss of the coherent oscillation of the free electrons over the volume of the particle. Increasing the size of the particle affects the plasmon resonances, with shifting resonance peaks to lower energies and broadening plasmon bands because of an increase in the radiation damping. ${ }^{[32,40]}$

The quasi-static approximation also becomes unreliable for very small particles. ${ }^{\text {[2] }}$ For nanoparticles smaller than the electron mean free path of the metal, elastic scattering at the surface of the particle increases the dephasing of the coherent oscillation of the electrons. This effect leads to a broadening in the resonance band width. For particles with even smaller dimensions in the range or below $1 \mathrm{~nm}$, quantum effects should be considered in order to describe their optical properties. ${ }^{[32]}$

\subsection{Plasmonic Structures}

There is a tremendous variety of plasmonic metal nanostructures and nanostructured surfaces, which can be distinguished according to the plasmonic modes they support: LSPRs or SPPs. ${ }^{[1,6,23]}$ The requirement for the structures that support LSPRs is to have dimensions smaller than the wavelength of light to experience a relatively uniform electric field when excited by light. Spherical nanoparticles are likely the most commonly used and well-studied type of nanostructures supporting LSPRs. ${ }^{[4]}$ Recent progress in nanoparticle synthesis has enabled designing plasmonic structures such as rods, disks, cubes, triangles, shells and stars, to support specific spectral resonance positions. ${ }^{[17]}$ Figure $2-5$ a to e, shows transmission electron microscopy (TEM) images of gold nanoparticles with different sizes and shapes and Figure 2-5f illustrates their corresponding absorption spectra, which shows the tunability of the absorption properties in gold nanoparticles by changing their geometry. ${ }^{[48]}$

One of the most widely used methods to create SPP waves at the interface of a flat metal film and a dielectric medium is to use prism coupling in Kretschmann or Otto configurations. ${ }^{[49]}$ SPPs can also be excited by using a periodic distribution of nanoridges or grooves in the metal's surface, for which the resonance condition depends not only on metal properties thickness and surrounding materials, but also on the grating periodicity and details of the nanostructures. ${ }^{[20,37]}$ Likewise, a convenient way to generate SPPs is via scattering from topographical defects on the surface, such as a subwavelength array of bumps or nanoholes. ${ }^{[34]}$ 

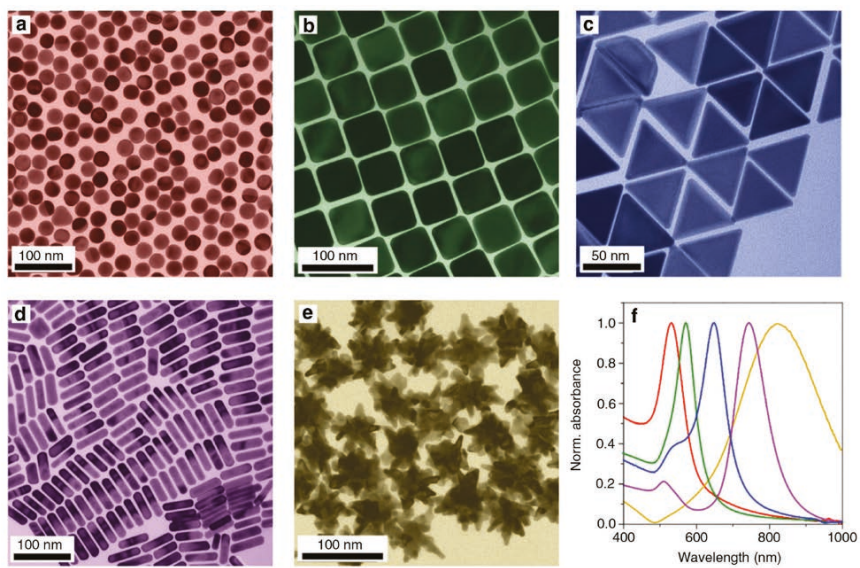

Figure 2-5. (a-e) TEM images of different gold nanoparticles. a) Nanospheres, b) Nanocubes, c) Nano-triangles, d) Nanorods, e) Nano-stars, f) Normalized absorption spectra of the nanostructures shown in the TEM images from a) to e. Reprinted with permission from reference [48].

Noble metal layers perforated with nanohole arrays can couple to incident light through SPPs and LSPRs, propagating on the continuous surface and localized inside the holes, respectively. ${ }^{[50]}$ SPP and LSPR wavelength is tunable by modifying the thickness and the diameter of the nanoholes.

In this thesis, gold nanodisk and nanohole arrays have been used in different devices for photothermal heating to harvest solar energy and for sensing of radiation. In the following sections, the optical properties of these structures will be discussed in more detail.

\subsubsection{Nanodisks}

Nanodisks are one of the most well-studied plasmonic structures. ${ }^{[15,51,52]}$ Their plasmonic resonance wavelength can be tuned from the visible to the near-infrared range by modifying the diameter $(d=2 a)$ or height $(2 c)$. Nanodisks are considered as oblate spheroids (Figure 2-6a) which have two axes of equal length $(a=b>c)$. Under the quasi-static approximation, where $c<\lambda$, the oblate spheroids are treated as discrete dipoles excited by an external field which is parallel to the major axis of the spheroid. ${ }^{[15,51]}$

The dipole polarizability $\alpha$ for such spheroid can be described as:

$$
\alpha=4 \pi a^{2} c \frac{\varepsilon-\varepsilon_{d}}{3 \varepsilon_{d}+3 L_{a}\left(\varepsilon-\varepsilon_{d}\right)} \quad(2-9)
$$


where $\varepsilon$ and $\varepsilon_{d}$ are the dielectric function of the spheroid (the metal) and the surrounding media, respectively, and $L_{a}$ is a geometrical factor that is a function of the aspect ratio of the nanodisk $(a / c) \cdot{ }^{[46]}$ Figure $2-6 \mathrm{~b}$ illustrates how increasing the aspect ratio of a nanodisk reduces the geometrical factor.

a)

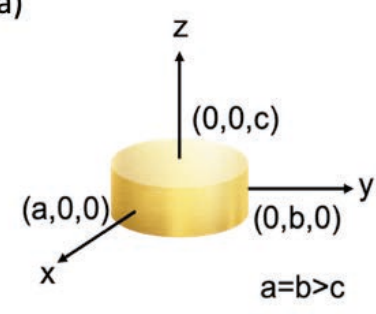

b)

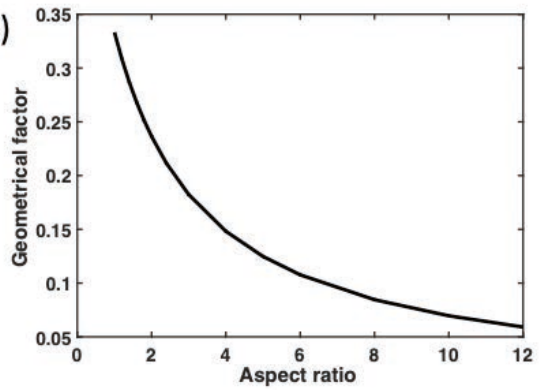

Figure 2-6. a) Schematic illustration of a gold nanodisk and its axes. b) The relation between the geometrical factor $\left(\boldsymbol{L}_{\boldsymbol{a}}\right)$ and aspect ratio $(\boldsymbol{a} / \boldsymbol{c})$.

To achieve the condition for plasmon resonance, the real part of the denominator in equation (2-9) should be equal to zero. The scattering, absorption and extinction cross-sections can be calculated using equations (2-6), (2-7), and (2-8), respectively. The plasmon resonance wavelength in a nanodisk is tunable by modifying the aspect ratio of the nanodisk. In the visible range where the dielectric function of gold can be described by $\varepsilon \approx k \lambda+m$, where $k$ and $m$ are real valued constants, the plasmon resonance wavelength can be calculated by $\lambda_{\max }=k^{-1}\left[\left(\varepsilon / L_{a}\right)+\varepsilon-m\right] .{ }^{\left[53,{ }^{54]}\right.}$ This shows that the plasmon resonance wavelength is inversely proportional to the geometrical factor and consequently depends on the aspect ratio of the nanodisk. For instance, increasing the aspect ratio of the nanodisk shifts the plasmon resonance to higher wavelengths. ${ }^{[33]}$

\subsubsection{Nanoholes}

In analogy to nanodisks, interaction of light with nanosized holes in noble metal films can also lead to pronounced optical resonances in the visible to near infrared spectral ranges. Interestingly, both LSPR and SPP modes can occur in metal nanohole systems, due to the localization of charges at the nanohole edges and through SPPs travelling on the continuous metal layer. ${ }^{[50,55]}$ 


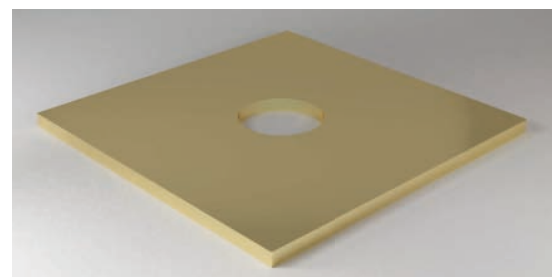

Figure 2-7. Illustration of a nanohole in a metal film.

To describe the optical properties of a single nanohole (Figure 2-7) in an optically thin metal film, it is necessary to consider both the diameter of hole and the thickness of metal film. Studies of the interaction of light with a single (gold) nanohole show that the hole plasmon resonance depends on the aspect ratio (diameter/height) of the hole. ${ }^{[25]}$ Increasing the aspect ratio shifts the hole plasmon resonance to longer wavelengths. This dependence is similar to that of nanodisks, although the underlying mechanisms are partly different. ${ }^{[55]}$ Nanoholes in optically thick metal films (a few hundreds of nanometers) strongly enhance the transmission of light, leading to extraordinary optical transmission which is not observed for the bare metal film. ${ }^{[22,56]}$ However, this behavior is not valid for an optically thin metallic film with and without nanoholes. In thin semitransparent metallic films, transmission reduces by introducing nanoholes in the film. ${ }^{[56]}$

Figure 2-8a illustrates simulated cases of these conditions, for $10 \mathrm{~nm}$ and $200 \mathrm{~nm}$ thick silver films perforated with arrays of nanoholes. The transmission peak for the optically thick perforated silver film and the transmission dip for the optically thin perforated film represent the plasmonic resonance positions in each film. As Figure $2-8 b$ shows, increasing the thickness of silver nanohole arrays from optically thin to optically thick layers leads to the formation of a dip-peak pair in the transmission spectra, which corresponds to only one resonance (positioned in between dip and peak) that is interfering with the continuum (direct transmission) state. This agrees with the Fano interference effect and makes it difficult to distinguish the correct positions of the plasmon resonances from transmission or extinction spectra of nanohole arrays. ${ }^{[56]}$ This matters specially in applications related to plasmonic heating, as studied in this work, for which absorptive plasmon resonances should be accurately designed and identified. Measuring the absorption spectra using an integrating sphere can help to determine the plasmonic resonance positions of such systems (will be discussed in detail in Chapter 4). ${ }^{[56]}$ 

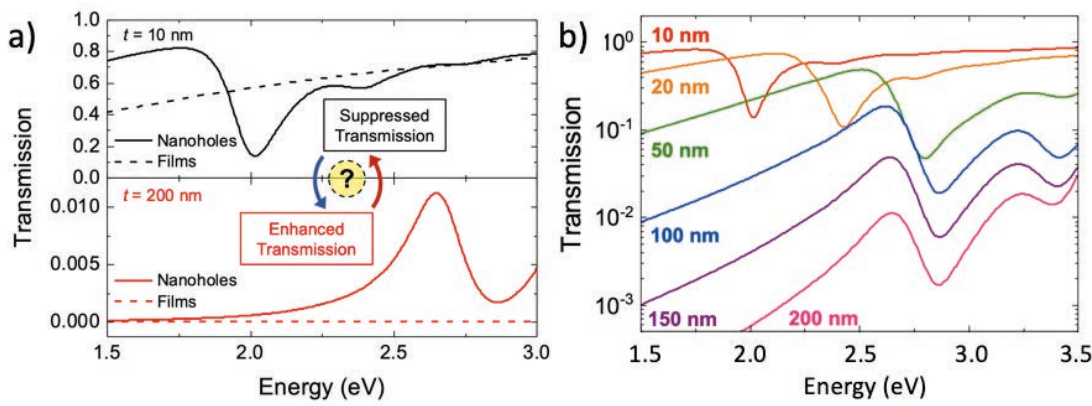

Figure 2-8. a) Transmission of light from a $10 \mathrm{~nm}$ silver film with nanohole array (solid black line) and without nanohole array (dashed black line) and from a $200 \mathrm{~nm}$ silver film with nanohole array (solid red line) and without nanohole array (dashed red line). b) Transmission spectra of various silver nanohole arrays with thickness from 10 to $200 \mathrm{~nm}$. Adapted with permission from reference [56].

\subsection{Plasmonic Properties of Ensembles}

Plasmonic resonances for a single particle mainly depend on the particle shape and size as well as the dielectric constant of the particle itself and the surrounding. ${ }^{[5]}$ In practice, using an ensemble of nanostructures is more common. The plasmonic resonances of periodic ensembles of nanoparticles or nanoholes are affected by the interaction between neighboring nanostructures. Near-field and far-field coupling are two types of interactions that govern the plasmonic resonances in an array of nanoparticles or nanoholes. ${ }^{[53]}$ Overlapping of the electromagnetic near-fields of metal nanostructures with very small interparticle spacing results in the generation of a new plasmonic modes with strongly enhanced electromagnetic near-fields between the structures. ${ }^{[53,57,58]}$ Nanostructures can also interact at longer distances via their far fields to form collective radiation, originating from the scattered fields by the nanostructures. Owing to a combination of near-field and far-field coupling, the plasmon lifetime and resonance wavelength strongly depend on the grating constant defined by the geometrical distribution of the nanostructures in the periodic arrays. ${ }^{[53}$, 58]

Fabricating long-range ordered plasmonic structures with high resolution often requires utilizing complicated, slow, energy consuming and expensive methods, such as electron beam lithography (EBL) and focused-ion beam lithography (FIB). ${ }^{[53]}$ For applications where perfectly ordered arrays are not necessary, short-range ordered arrays can be a suitable alternative, which can be fabricated by relatively cost-effective and simple methods such as colloidal lithography (discussed in more detail in Chapter 
4). ${ }^{[59-61]}$ This method is specifically suitable to rapidly produce large areas $\left(>\mathrm{cm}^{2}\right)$ of nanostructures (such as disks or holes) distributed in short-range order. ${ }^{\left[60,{ }^{61]}\right.}$ Shortrange ordered nanostructures are not spread randomly, but there is a characteristic distance between neighboring nanostructures, which can be controlled during the fabrication process. ${ }^{[62]}$ For arrays with a short-range order larger than a few tens of nanometers, the effect of near-field coupling is minimized. The lack of nearfield and far-field coupling in these structures makes their optical properties less dependent on interparticle spacing and more reliant on the single particle properties. ${ }^{[53]}$ The plasmon resonance wavelength for short-range ordered nanodisks is known to be almost independent on the interparticle spacing. ${ }^{[53]}$ On the contrary, reducing the interparticle spacing in a short-range ordered nanohole array blue shifts the plasmonic wavelength, which indicates a larger inter-hole coupling through the metal film for short-range ordered nanoholes compared with discrete structures distributed in the same manner. ${ }^{[53,62]}$

\subsection{Physics of Plasmonic Heating}

Noble metal nanostructures interact strongly with light via the excitation of surface plasmons. The plasmons decay through radiative and non-radiative channels, which represent scattering and absorption, respectively. ${ }^{[15,32,63,64]}$ The non-radiative damping is attributed to the electronic structure of the metal through its dielectric function, which has contributions from both intraband (electron scattering processes inside the nanoparticle) and interband excitations. ${ }^{[15]}$ As it is clear from equations 2-5 to 2-7, absorption and scattering cross-sections are related to the polarizability and size of nanostructure. The absorption cross-section increases slower with particle size than the scattering cross-section. ${ }^{[9]}$ This relation makes small plasmonic particles primarily absorptive, while larger particles are more efficient scatterers. ${ }^{[9]}$

For nanoparticles with negligible quantum yield (i.e. they are very weak light emitters), there is a direct relation between the amount of light that is absorbed by a nanoparticle (absorption cross-section) and the amount of heat that is generated $(Q)$ in the nanoparticle. This can be expressed as: ${ }^{[65,66]}$

$$
Q=I \sigma_{a b s} \quad(2-10)
$$

where $I$ is the irradiance of the incident light (power per unit area). The generated heat can also be separated into the heat power density $q(r)$ inside the nanoparticle as: $:^{[66,67]}$

$$
Q=\int_{V_{N P}} q(r) d^{3} r \quad(2-11)
$$


where the integral runs over the nanoparticle volume $\left(V_{N P}\right)$. On the other hand, the mechanism of plasmonic heating can be described by the Joule effect, originating from the optically-induced current in the metal as: ${ }^{[9]}$

$$
q(r)=\frac{1}{2} \operatorname{Re}\left[J^{*}(r) \cdot E(r)\right] \quad(2-12)
$$

where $J^{*}(r)$ is the complex amplitude of the current density and $E(r)$ is the inner electric field created in the particle due to the plasmonic excitation. This equation can be re-written as: ${ }^{[9]}$

$$
q(r)=\frac{\omega \varepsilon}{2} \operatorname{Im}(\varepsilon(\omega))|E(r)|^{2} \quad(2-13)
$$

which shows that there is a direct relation between the heat generation density inside the nanoparticle and the imaginary part of the dielectric constant of the metal nanoparticle and the square of the inner electric field amplitude. ${ }^{[9]}$

The power generated in nanoparticles with simple geometries can be calculated analytically using their cross-section expressions using equations $2-6$ to $2-8$ and known polarizabilities (for a nanosphere, it can be derived from equation 2-5). ${ }^{[9,66]}$ For more complicated geometries, for which there are no straightforward analytical expressions for the absorption cross-section, the heat generated in the nanoparticle can be obtained by optical simulations, either by directly providing the optical crosssection or by computing the inner electric field amplitude followed by calculating the absorption using equations (2-10, 2-11 and 2-12). ${ }^{[9]}$ Examples of simulation techniques that can be used to simulate the optical response are the finite-difference time-domain (FDTD) and the finite element methods (FEM). ${ }^{[68,69]}$ The amplitude of the electric field inside a nanoparticle can also be investigated numerically and quantitatively using Green's dyadic method (GDM). ${ }^{[66]}$ This method helps to understand the thermal mapping in nanoparticles with complicated geometries.

The temperature distribution inside and outside a single spherical nanoparticle of radius $(R)$ at different distances from the particle center $(r)$ can be described by:[9]

$$
\begin{aligned}
& \delta T(r)=\delta T_{N P} \frac{R}{r}, \quad r>R \text { (outside particle) } \quad(2-14) \\
& \delta T(r) \approx \delta T_{N P}, \quad r<R \text { (inside particle) } \quad(2-15)
\end{aligned}
$$

where $\delta T_{N P}$ is the temperature increase inside the nanoparticle upon illumination. Due to the uniform dissipation of heat in all directions from the sphere, $\delta T$ decreases inversely with distance from the particle. For a spherical nanoparticle, $\delta T_{N P}$ is related to the absorbed power and inversely proportional to the thermal conductivity of the surrounding medium $\left(k_{s}\right)$ according to: ${ }^{[9]}$

$$
\delta T_{N P}=\frac{Q}{4 \pi k_{s} R} . \quad(2-16)
$$


Inside a nanoparticle the heat power density $(q(r))$ can be quite non-uniform, however, the temperature remains quasiuniform due to the high thermal conductivity of the metal compared with that of the surroundings. ${ }^{[9]}$

The steady state temperature of the particle is dependent on the amount of absorbed heat, the particle size, and the thermal conductivity of the surrounding medium. The heat generated by the nanoparticle and the thermal conductivity of the surrounding medium determine the temperature elevation of the surrounding medium through plasmonic heating by the plasmonic nanoparticle. ${ }^{[9]}$

\subsubsection{Plasmonic Heating in Ensembles of Gold Nanodisks or Gold Nanoholes}

Plasmonic heating of an individual nanoparticle such as a nanodisk can be considerably enhanced by the presence of nearby nanoparticles. If those nearby nanostructures are also illuminated, the heat dissipated from them can contribute significantly to the temperature increase of the nanoparticle. Hence, plasmonic nanostructures in ensembles heat up each other through collective effects. ${ }^{[0]}$ The average temperature increase of such plasmonic arrays highly depends on particle density in the ensemble, that can be interpreted as the density of heat sources. ${ }^{[9,66]}$

Discrete plasmonic structures like nanodisks have been widely used in thermoplasmonic systems as point-like heat sources, due to their high photothermal heating efficiency, tunability of their absorption resonance wavelength, narrow peak width, and possibility to use them in solution. ${ }^{[10,30,53,71,72]}$ On the contrary, continuous plasmonic structures, such as perforated metal layers, have been mostly utilized as heat sinks to minimize plasmonic heating, by effectively dissipating heat generated from a small area illuminated by focused light. ${ }^{[73]}$ However, for uniform illumination of larger arrays, the metal film can no longer act as a heat sink. For such conditions, plasmonic nanoholes systems can generate higher temperature increase compared to discrete plasmonic structures. ${ }^{[74]}$ This was recently shown through a comparison between optically thin gold nanohole arrays and gold nanodisk arrays of the same dimensions. The gold nanohole arrays absorbed more light than the nanodisk arrays at shorter wavelengths since they contain more metal and provide higher material absorption due to interband transitions. The nanohole arrays also absorbed more light than a non-perforated gold film due to plasmon excitation at longer wavelengths and associated absorption losses. ${ }^{[74]}$ From an application point of view, it should also be noted that high electrical and thermal conductivity of metal nanohole structures allow them to provide thermoplasmonic systems with electrical contacts for collecting 
signals and rapid and uniform heating, respectively. These properties make metal nanohole arrays good candidates for use as broad-range absorbers in thermoplasmonics systems, such as for applications that uses broad-band light source like the sun. ${ }^{[74]}$ 


\section{Chapter 3: Thermal Energy Harvesting and Sensing}

Temperature is one of the most familiar physical variables and it plays a vital role in controlling and affecting a broad range of processes in organic biological systems as well as in inorganic systems. ${ }^{[75,76]}$ The first attempts of humans to control temperature for specific application dates back to the bronze and iron ages when they used fire to make tools out of those metals. For thousands of years, human beings have been developing ways to measure and control temperature. One of the very preliminary methods that are still used to measure temperature is based on the thermal expansion of materials such as mercury. ${ }^{[75]}$ New methods and approaches have been developed to address the need for more accurate and quick temperature evaluation of different systems, for use in diverse applications such as controlling temperature-dependent chemical reactions, fire alarms and food processing. ${ }^{[75,76]}$

Biological systems utilize a sophisticated sensory system to detect variations in temperature to interact with the outside world. ${ }^{[76-80]}$ Some types of animals such as snakes, ${ }^{[81]}$ frogs, ${ }^{[82]}$ and fishes ${ }^{[82]}$ can also detect thermal radiation from a warm object, which enables them to navigate and hunt in murky environments. Our skin also enables us to sense heat from both touching a warm object and absorbing light radiation. ${ }^{[83-86]}$ Attempts to mimic such fascinating system have led to developing a platform technology called electronic skin or e-skin. ${ }^{\text {[87-91] }}$

Heat flux and thermal radiation sensing in e-skin concepts can give the sense of touch and heat of sun light to prosthetic limbs and enables remote healthcare monitoring. ${ }^{[83,84,89,92-94]}$ For e-skin applications, it is essential to reduce the bulkiness and power consumption of the functional systems through designing self-powered sensors that can detect various stimuli. ${ }^{[95,96]}$ Thermal sensitive materials that generate 
electric response upon temperature variations can play an effective role to accomplish such goals. Moreover, the ingratiation of these functional materials with plasmonic structures in a hybrid system can enable sensing temperature variations from direct heating and radiation heating.

Hybrid systems also enable harvesting energy from heat. Temperature gradients and heat fluxes are considered as environmental energy sources that can be converted directly to electricity using pyroelectric and thermoelectric systems. ${ }^{[97-99]}$ These sources of thermal energy can range from the heat of human body for energizing wearable electronics, to solar light for hybrid thermoelectric generators. ${ }^{[100,101]}$ This chapter introduces materials and means that were employed in this thesis to convert temperature variations from direct heating and photothermal heating into an electric signal for energy harvesting and sensing. Then, it discusses thermal-induced charging processes as another method to harvest thermal energy from heat sources with low temperatures $\left(<250^{\circ} \mathrm{C}\right)$. The chapter ends with a discussion on different types of temperature sensors.

\subsection{Heat-Induced Electric Potential Generation in Materials}

\subsubsection{The Thermoelectric Effect}

Thermoelectrics describes the Seebeck effect and the Peltier effect resulting from coupling between heat transfer and electricity in conductor and semiconductor materials. ${ }^{[102]}$ The Seebeck effect was discovered in 1821 by Thomas Johann Seebeck, who observed that a temperature difference $(\Delta T)$ between two junctions of dissimilar materials (a thermocouple (TC)) induces an electric potential $(\Delta V)$ across the junctions, which is proportional to the temperature difference and can be described by the Seebeck coefficient $(S) .{ }^{[103,104]}$ The Seebeck coefficient is an intrinsic property of materials and describes the ratio of the established voltage for a given temperature difference $(\Delta V / \Delta T)$ across the material. ${ }^{[105]}$ Generally, a material can have a negative or a positive Seebeck coefficient depending on the type of the majority of charge carriers in the material, that can be electrons or holes. ${ }^{[106-108]}$ In a conductor with a negative Seebeck coefficient, electrons as the majority charge carriers migrate from the hot side to the cold side of the conductor subjected to a temperature gradient. Hence, it is established an electron-density gradient and a corresponding electric potential across the hot and cold ends of the conductor. ${ }^{[108,109]}$

The Seebeck effect is the working principle of thermoelectric generators (TEGs), which are solid-state energy harvesting devices that can convert static temperature 
gradients to electricity without any moving parts. Figure 3-1a illustrates the structure of a typical TEG, consisting of a pair of p-type and n-type thermoelectric legs. These legs are connected by metal electrodes such that they are electrically in series and thermally in parallel. ${ }^{[10]}$ A temperature gradient over the $\mathrm{p}$ and $\mathrm{n}$ legs leads to thermodiffusion of charge carriers from the hot side to the cold side, which induces a thermoelectric potential and can drive a current through a load in an external circuit. ${ }^{[10]}$

In 1834, Jean Peltier observed that flowing an electric current through the junction of dissimilar materials establishes a temperature difference between the junctions and interestingly the temperature increase at the junction with higher temperature could not be explained by Joule heating alone. He also observed that the temperature of the junction could increase or decrease depending on the electric current direction. The phenomenon was later termed the Peltier effect, which is the working principle of Peltier coolers. ${ }^{[105]}$ Figure 3-1b schematically depicts the structure of a Peltier cooler, which is analogous to the TEG. Applying an electric potential to the legs of a Peltier cooler leads to the movement of charge carriers and generation of a temperature gradient over the legs.
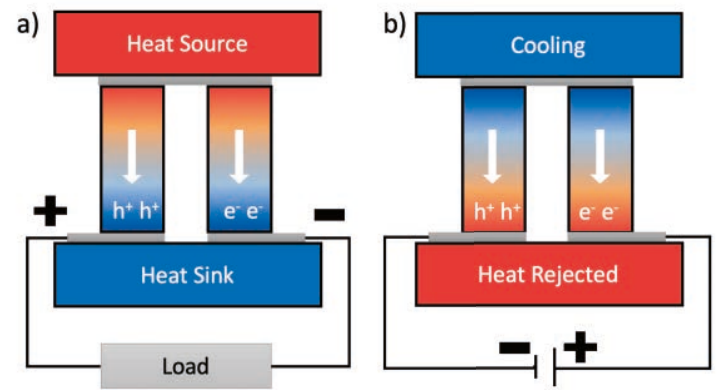

Figure 3-1. Schematic illustration of thermoelectric generators. a) A thermoelectric generator consisting of a pair of p-type and n-type materials which their ends are kept in cold and hot condition. It generates electric potential over the connected load. b) A Peltier cooler with similar structure as in (a) connecting to a power supply.

\subsubsection{The Soret Effect}

A temperature gradient over a material consisting of two or more components can act as a driving force for mass diffusion. This so-called thermodiffusion effect, or Soret effect, occurs in gases, liquids and even some solid systems. ${ }^{[111,112]}$ Figure $3-2 \mathrm{a}$ illustrates the concentration gradient $(\nabla c)$ of particles in a diluted colloidal 
suspension subjected to a temperature gradient ( $\nabla T)$, where the heat and the concentration gradients affect the mass flux of particles $(J):^{[112]}$

$$
J=-c D_{T} \nabla T-D \nabla c . \quad(3-1)
$$

where $D_{T}$ and $D$ are thermodiffusion coefficient and diffusion coefficient, respectively. In the steady state condition where the mass flux $(J)$ is zero, the concentration gradient is constant and can be read as:[112]

$$
\nabla c=-c S_{T} \nabla T \quad(3-2)
$$

in such condition, the magnitude of thermodiffusion, the Soret coefficient $\left(S_{T}\right)$, is determined by the ratio of the thermodiffusion coefficient and the diffusion coefficient as: $:^{[112]}$

$$
S_{T}=\frac{D_{T}}{D} . \quad(3-3)
$$

The sign of $S_{T}$ determines if the particle drifts preferentially towards colder or hotter regions. ${ }^{[13]}$ For systems with $S_{T}>0$, the particles drift towards the cold side, and if $S_{T}<0$, the particles accumulate on the hot region. ${ }^{[13]}$ The sign and magnitude of the thermodiffusion depend on the specific properties of the system such as surface charge of the particles, particle size and concentration, and the interaction between particles and solvent. ${ }^{[111,113,114]}$

In electrolytes and other liquid systems containing positive and negative charged particles (cations and anions, respectively), the Soret effect results in a concentration gradient of charged particles (Figure 3-2b) along the temperature gradient $(\Delta T)$ and consequently an internal electric field $(E)$ across the material. ${ }^{[15,116]}$ This electric field is manifested as an open circuit electric potential $\left(V_{o c}\right)$ generated by a system containing a non-redox-active electrolyte subjected to a temperature gradient and sandwiched between two electrodes. ${ }^{[116]}$

a)

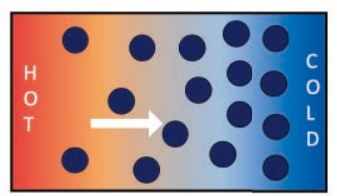

b)

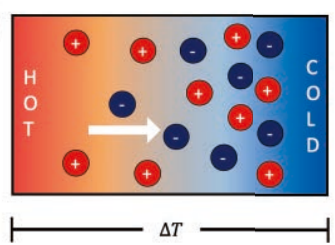

Figure 3-2. Schematic illustration of thermodiffusion of particles in a diluted colloidal suspension (a), and thermodiffusion of positive and negative ions in an electrolyte (b) subjected to a temperature gradient. 
In analogy to conventional inorganic thermoelectric materials, the ionic Seebeck coefficient of the electrolyte can be derived as $S_{i}=V_{o c} / \Delta T \cdot{ }^{[16]}$ Hence, these systems can be used as a type of ionic thermoelectric materials.

The open circuit voltage generated by such system depends on the temperature difference and the profiles of the concentration gradients of both cations and anions across the electrolyte. ${ }^{[16]}$ In turn, these concentration gradients rely upon the diffusion and Soret coefficients of cations and anions as well as the concentration of dissociated ions. ${ }^{[116]}$

\subsubsection{Thermoelectric Materials}

The thermoelectric properties of materials are commonly evaluated by their dimensionless figure of merit $(Z T)$, defined as: $:^{[102]}$

$$
Z T=\frac{S^{2} \sigma T}{k} \quad(3-4)
$$

where $\sigma$ and $k$ are the electrical and thermal conductivity, respectively, and $T$ is the temperature. According to $Z T$, materials can provide maximum efficiency in TEGs only if they have a high electrical conductivity to contain sufficient amount of charge carriers, a high Seebeck coefficient to produce large electric potential, and a minimized thermal conductivity to sustain a large temperature difference over the material. However, it is challenging to achieve that high $Z T$ due to the interdependency of these three parameters. ${ }^{[102]}$

The Seebeck coefficient and electrical conductivity have an inverse dependency on the charge carrier (electron and hole) concentration, so that increasing the charge carrier concentration in a material reduces the former and increases the latter. ${ }^{[102,110]}$ Moreover, two sources contributing to heat transfer in materials are charge carriers transporting heat and phonons travelling through the lattice. ${ }^{[101,117]}$ Therefore, increasing charge carrier density has a positive effect on the thermal conductivity of the material. After all, to achieve a high thermoelectric power factor $\left(S^{2} \sigma\right)$ and consequently a high $Z T$ in a material, one parameter or more should be enhanced while preventing the deterioration of the others. ${ }^{[101,110,117]}$ This has led to the design and production of various materials and structures, some of which are presented in Figure 3-3. This figure outlines approximate regions for the Seebeck coefficient and the electrical conductivity of various thermoelectric materials.

A wide variety of semiconductors and semimetals, such as metal alloys, ceramics, conducting polymers and their derivatives have been extensively explored for thermoelectric applications. ${ }^{[101,110]}$ 


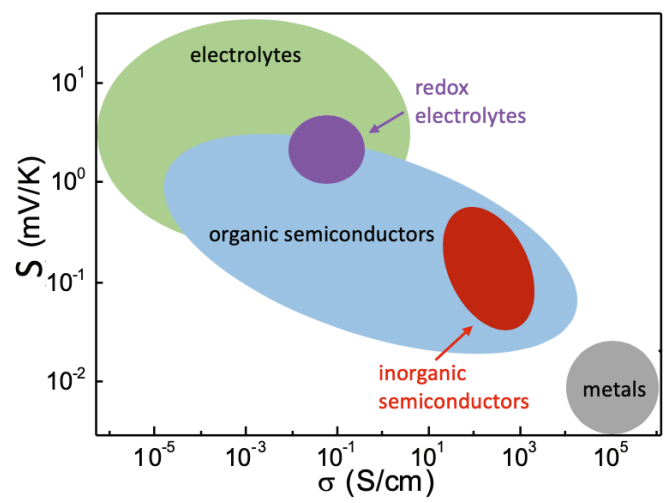

Figure 3-3. Approximate ranges for the Seebeck coefficient as a function of electrical conductivity of some common types of thermoelectric materials. ${ }^{[116,118-120]}$

Another class of materials that have similar conductivity to semiconductors but higher Seebeck coefficient are redox-active electrolytes (purple area) and non-redoxactive electrolytes (green area). While these ionic thermoelectric materials show a relatively low conductivity, their high Seebeck coefficients make them suitable candidates for non-conventional thermoelectric applications such as heat sensing. ${ }^{[116,}$ 121, 122]

Redox-active electrolytes are used in thermogalvanic cells, in which an oxidationreduction reaction occurs between the electrodes and the electrolyte as long as a temperature gradient exists between the two electrodes. In these types of cells, ions circulate within the cell and exchange electrons with electrodes. ${ }^{[123]}$ On the other hand, non-redox-active electrolytes, such as some ionic liquid electrolytes, are inert electrolytes that can be used in thermoelectric devices without involving any chemical reactions, ${ }^{[123]}$ as the one chosen for this work.

\subsection{Ionic Liquids and Their Derivatives for Use in Thermoelectric}

\section{Applications}

More than a century has passed since Paul Walden in 1914 was searching for molten salts for his equipment, which led to the discovery of ionic liquids (ILs). ${ }^{[124]}$ Ionic liquids generally consist of organic cations and organic or inorganic anions with melting point below $100{ }^{\circ} \mathrm{C} .{ }^{[125]}$ The bulkiness of the compounds in ILs leads to weak ionic bonds, because the ions are separated and the ionic strength is mainly based on the Coulomb force that decreases in strength with distance. ${ }^{[124]}$ 
a)

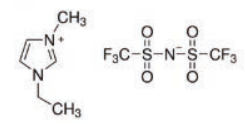

b)

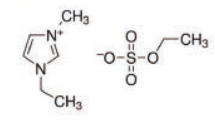

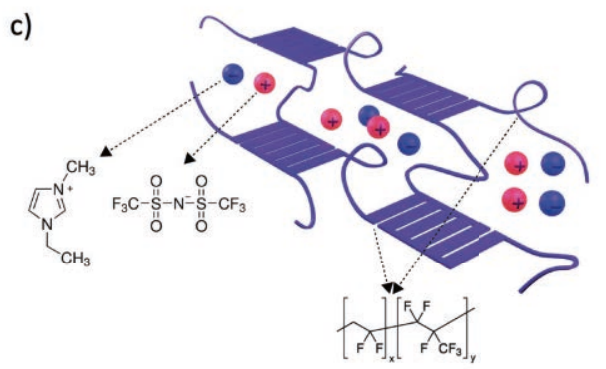

Figure 3-4. Chemical structure of a) 1-ethyl-3-methylimidazolium ([EMIM]) bis(trifluoromethylsulfonyl)imide ([TFSI]) and b) 1-ethyl-3-methylimidazolium ethylsulfate $[\mathrm{EMIM}]\left[\mathrm{ESO}_{4}\right]$. c) Schematic illustration of the polymer gel composition and structure. ${ }^{[16]}$

Ionic liquids have attracted significant attention due to their interesting properties, such as low vapor pressure, high ionic conductivity, high thermal stability, ability to dissolve various type of materials, non-toxicity and environmental friendliness compared to hazardous volatile organic solvents. ${ }^{[118,124,126]}$ ILs are furthermore referred to as "designer solvents" owing to the wide variety of cation-anion combinations that can be designed and used to tune the polarity, hydrophilicity, and hydrophobicity for use in specific applications. ${ }^{[124]}$ Despite all positive properties that ILs have, the sensitivity and physical properties of some types tend to change by moisture and oxides impurities. ${ }^{[124]}$ This introduces some limitations such as the necessity of providing an inert atmosphere or using glovebox in the process of fabrication and usage of the samples containing ILs. A group of ILs, such as 1-ethyl-3methylimidazolium $\left(\right.$ EtMeim $^{+}$) salts, was designed with more stable properties against humidity, which allows broad usage of ILs as electrolytes in different environments. ${ }^{[127]}$

1-ethyl-3-methylimidazolium ([EMIM]) bis(trifluoro-methylsulfonyl)imide ([TFSI]) and 1-ethyl-3-methylimidazolium ethylsulfate [EMIM][ESO $\left.{ }_{4}\right]$ are two specific types of EtMeim ${ }^{+}$salts that we used in the work of this thesis. Figure 3-4a and b show the chemical structure of their consisting anions and cations, respectively. ${ }^{[116,124]}$

ILs have relatively high Seebeck coefficients (about $0.35 \mathrm{mV} \mathrm{K}^{-1}$ for [EMIM] [ $\left.\mathrm{ESO}_{4}\right]$ ) and typically low thermal conductivities (about $0.18 \mathrm{~W} \mathrm{~m}^{-1} \mathrm{~K}^{-1}$ at room temperature

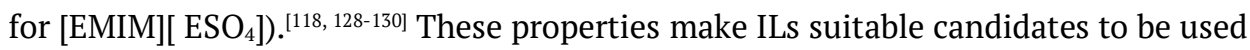
in the TEGs and temperature sensors for low-grade temperature harvesting and 
sensing applications in wearable electronics or e-skin. ${ }^{[128]}$ However, utilizing these materials in thermoelectric devices is challenging because the devices require proper sealing and encapsulation due to the liquid phase of these electrolytes. On the contrary, polymer-based electrolytes are more attractive since they can be made as free-standing gels while showing a high Seebeck coefficient. ${ }^{[18]}$ For temperature sensing applications based on the Seebeck effect, it is preferable to use a material with a high Seebeck coefficient and a low thermal conductivity. ${ }^{[131]}$ Using such material enables generating higher electric signals in response to a defined temperature change compared to a material with lower Seebeck coefficient and higher thermal conductivity at the same condition. ${ }^{[131]}$

In this thesis, a composite thermoelectric gel (Figure 3-4c) was used, containing copolymer poly(vinylidene fluoride-co-hexafluoropropylene) (PVDF-HFP) as a solid matrix and [EMIM][TFSI] as the electrolyte (from now on, it is named as ionic thermoelectric gel), with a negative Seebeck coefficient of -4 to $-5 \mathrm{mV} \mathrm{K}^{-1}{ }^{\text {. }{ }^{[132]} \text { The }}$ negative sign of the Seebeck coefficient comes from dissociated anions that thermodiffuse towards the colder side of the gel, when it is subjected to a temperature gradient. The thermodiffusing ions in ionic thermoelectric materials cannot pass into an external circuit of a TEG, instead they accumulate at the interface of the metal electrode and the ionic thermoelectric material.[116] Similar to a charge storage process, this accumulation process induces an electric current in the external circuit that decays over time. This concept can be used to charge supercapacitors (discussed in section 3.2) or batteries by increasing the accumulated charge density using electrode materials with high ionic conductivity and large surface area. ${ }^{[116,119,133]}$ In 2016, Zhao et al. ${ }^{[133]}$ reported the conversion of heat into stored charge in a so-called ionic thermoelectric supercapacitor, consisting of a polymeric electrolyte with high a Seebeck coefficient $\left(10 \mathrm{mV} \mathrm{K}^{-1}\right)$ working as the ionic thermoelectric material and carbon nanotubes as electrodes with high surface area. The stored energy can later be used to power an external circuit whenever it is needed. ${ }^{[133]}$

A typical thermoelectric generator needs both n-type and p-type thermoelectric materials that are connected electrically in series and thermally in parallel. In 2019, Zhao et al. ${ }^{[116]}$ showed an ionic thermoelectric generator consisting of thermoelectric gels with negative and positive Seebeck coefficients, working analogous to the semiconductor thermoelectric generator. ${ }^{[116]}$ One attractive feature of this ionic thermoelectric generator is the possibility of low-cost mass production by roll-to-roll printing, making the concept interesting also for use as high-resolution temperature sensors for flexible and large-area applications such as e-skin. ${ }^{[116]}$ 


\subsubsection{The Pyroelectric Effect}

Pyroelectricity is another phenomenon that is commonly used to convert heat stimuli to electric signals. Any material with polar point group crystal structure can show the pyroelectric effect, for which a change in temperature over time alters the spontaneous electrical polarization of the material. ${ }^{[34-137]}$ The change in the spontaneous polarization with temperature is described by a vector called the pyroelectric coefficient $(p)$ that under constant electric field $(E)$ and stress $(\sigma)$, can be defined as: $:^{[138]}$

$$
p=\left(\frac{\partial P_{S}}{\partial T}\right)_{\sigma, E} \quad(3-5)
$$

where $P_{S}$ is the magnitude of the electric polarization vector. A polar pyroelectric material sandwiched between two electrodes, with $P_{S}$ perpendicular to the electrode surfaces, can be considered as a capacitor that can store screened charges $(Q)$ on the electrodes. ${ }^{[136,137]}$ The internal arrangement of the electric dipoles in the pyroelectric material varies with a change in temperature, through reorientation of the molecules or displacement in the atomic arrangement. The temporal change in temperature and corresponding changes in the spontaneous polarization cause a variation in the screened charge on the electrodes. The exceeding surface charge can flow into an external circuit as a transient pyroelectric current $\left(i_{p}\right)$ given by:[135-137, 139]

$$
i_{p}=\frac{\mathrm{d} Q}{\mathrm{~d} t}=A p \frac{\mathrm{d} T}{\mathrm{~d} t} \quad(3-6)
$$

where $A$ is the electrode surface area, and $\mathrm{d} T / \mathrm{d} t$ is the rate of temperature change over time. Thus, pyroelectric devices can be responsive to any energy input that generates a temperature change to the pyroelectric element. ${ }^{[135,136]}$ The pyroelectric effect has been known for many years and has since about 1960 been used in fire alarms and for electromagnetic radiation detection in thermal imaging devices, as well as for pollution monitoring and gas analysis. ${ }^{[135,136]}$

Ferroelectric crystalline polar dielectric materials provide large pyroelectric coefficients, which is related to their lack of inversion symmetry and strong spontaneous polarization due to the difference in the electronegativity of the building atoms. ${ }^{[140]}$ These materials show spontaneous polarization up to a certain temperature. Above a critical temperature called the Curie temperature $\left(T_{c}\right)$, an orderdisorder phase transition occurs, which disturbs the spontaneous polarization such that the material loses its pyroelectric properties. ${ }^{[141]}$ The resulting polycrystalline material has randomly assembled domains with dipoles oriented in different directions and therefore does not provide the net polarization necessary for pyroelectricity. ${ }^{[141]}$ Through a poling process, applying an electric field greater than 
the so-called coercive field over the material rearranges the polar axes of the domains and aligns them along the applied electric field to restore the net spontaneous polarization and hence the pyroelectric effect. ${ }^{[135,136]}$ Ferroelectric materials can maintain their net spontaneous polarization even after removing the applied external electric field. ${ }^{[142]}$ Ferroelectric materials possess piezoelectric properties apart from pyroelectric properties, which enable them to also convert mechanical stimuli to electrical signals and vice versa. ${ }^{[142]}$

\subsubsection{Pyroelectric Materials and Their Applications}

There is a wide variety of intrinsic pyroelectric materials in the form of single crystals, ceramics, and polymers that are suitable for specific applications and working temperatures depending on their $T_{c}$, mechanical and chemical properties. ${ }^{[135}$, ${ }^{136]}$ Although the pyroelectric coefficients of polymer pyroelectrics are lower than that of the inorganic pyroelectric materials, such as the well-known lead zirconate titanate (PZT), organic pyroelectrics have become popular for different applications due to their mechanical flexibility as well as their inexpensive and low temperature fabrication processes. ${ }^{[143]}$ Among all, ferroelectric polymer materials such as poly(vinyl chloride) (PVC) and Nylon 11, poly(vinylidene fluoride) (PVDF) and its copolymer, poly(vinylidenefluoride-co-trifluoroethylene) ( $\mathrm{P}(\mathrm{VDF}-\mathrm{TrFE})$ ), have attracted much attention for use in pyroelectric applications lower than $100{ }^{\circ} \mathrm{C} .{ }^{[144-146]}$ These materials can be deposited and patterned in the form of pyroelectric arrays on different substrates.

PVDF is one of the most promising ferroelectric polymer thanks to its high chemical stability, non-toxicity, highly compact structure, and relatively large permanent dipole moment. Polar vinylidene fluoride monomers $\left(-\left[\mathrm{CH}_{2}-\mathrm{CF}_{2}\right]^{-}\right)$are elementary repeating units in PVDF. The polar property in PVDF originates from the large difference in electronegativity between fluorine, carbon and hydrogen atoms, creating a dipole moment of 2.1 Debye across the monomer. ${ }^{[147]}$

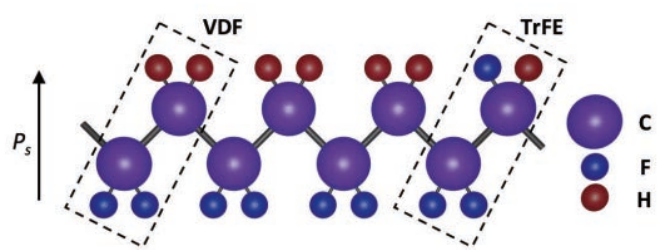

Figure 3-5. Schematic illustration of the molecular structure of $\mathrm{P}(\mathrm{VDF}-\mathrm{TrFE})$ and the polarization direction pointing from fluorine to hydrogen atoms. ${ }^{[148]}$ 
Typically, PVDF has low crystallinity and it is polymorphous, consisting of both an amorphous phase and one or more crystalline phases. ${ }^{[147]}$ Ferroelectricity and pyroelectricity of PVDF originate from the presence of the so-called $\beta$ phase, which is a minority phase in this material. Obtaining the $\beta$ phase in PVDF needs relatively complicated procedures, such as electrical poling combined with mechanical stretching. ${ }^{[14]}$

The presence of PTrFE in the copolymer P(VDF-TrFE) (Figure 3-5) enhances the crystallinity such that thin films with more than $90 \%$ crystallinity can be obtained from simple solution processing methods such as spin coating followed by thermal annealing at $130^{\circ} \mathrm{C} \cdot{ }^{[147]}$

As prepared solvent-formed $\mathrm{P}(\mathrm{VDF}-\mathrm{TrFE})$ copolymer film consists of $\beta$ phase polycrystalline grains and amorphous regions. In such film, the polarization of different domains (areas with similar polarization) cancels out each other, leading to a nearly zero residual spontaneous polarization over the film (Figure 3-6a). To prepare a polarized film, it is necessary to obtain domains with similar polarization direction, which is attainable through a so-called poling process. ${ }^{[139,149]}$

Figure 3-6b shows the electrical current as a function of applied electric field over a P(VDF-TrFE) film sandwiched between two metallic electrodes. This figure shows that for applied electric fields lower than the coercive field $\left(100 \mathrm{~V} \mathrm{\mu m}^{-1}\right)$ the current is low. Applying an electric field larger than the coercive field establishes an electric current owing to the alignment of the dipoles and corresponding changes of the surface charge on the electrodes.

a)

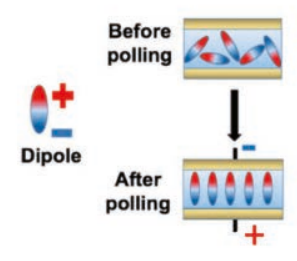

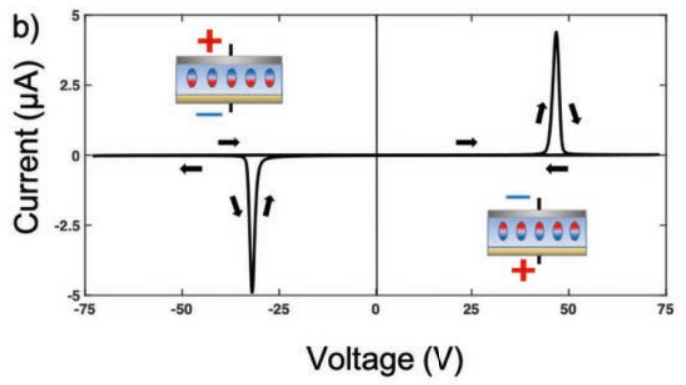

Figure 3-6. a) Schematic illustration of dipoles in a P(VDF-TrFE) film sandwiched between two metal electrodes before (top) and after (bottom) poling process. b) Cyclic voltammetry of $\mathrm{P}(\mathrm{VDF}-\mathrm{TrFE})$ copolymer film presented in (a) which is showing the repolarization current as a function of applied voltage. The inset shows switching of the dipoles in the P(VDFTrFE) film by changing the sign of applied voltage. Adapted with permission from reference [149]. 
The current reduces to lower values when all dipoles are aligned with the applied electric field. The absence of an electric current when reducing the applied electric field to zero again indicates that the dipoles stay aligned. Hence, there is a residual spontaneous polarization over the $\mathrm{P}(\mathrm{VDF}-\mathrm{TrFE})$ film. The alignment of the dipoles and the polarization remain unchanged until an electric field is applied, which is as large as the coercive field with opposite polarity.

The ferroelectric, pyroelectric, and piezoelectric properties of $\mathrm{P}(\mathrm{VDF}-\mathrm{TrFE})$ have made this material a suitable candidate for nonvolatile memory applications, temperature and radiation sensors, tactile sensors, strain and acceleration measurement devices to name but a few. ${ }^{[143]}$ Another interesting and fast-growing area for using $\mathrm{P}(\mathrm{VDF}-\mathrm{TrFE})$ is energy harvesting. Although the first attempts to use P(VDFTrFE) for converting heat to electricity in 1963 by Hoh and Beam et al. ${ }^{[150]}$ resulted in very low conversion efficiency, theoretical work by Drummond and Gonzalo suggested that much higher efficiency might be achievable. ${ }^{[151]} \mathrm{A}$ simple approach to harvest energy from a pyroelectric material such as $\mathrm{P}(\mathrm{VDF}-\mathrm{TrFE})$ copolymer film is to connect it to an electrical resistor and apply a temperature change. ${ }^{[152]}$ The change in temperature with time generates an electrical current through the resistor. ${ }^{[152]}$ The output power generated by such system enhances with increasing amplitude and frequency of the temperature changes and using materials with high pyroelectric coefficient. ${ }^{[152]}$ The pyroelectric response of a pyroelectric capacitor subjected to a temperature change is fast, but the generated signal is transient and it decays as the pyroelectric element reaches a temperature equilibrium. Hence, these systems cannot provide energy at constant temperatures, but provide means to harvest energy from changes in temperature. ${ }^{[152]}$

The transient signal upon temperature changes can also be used for sensing applications. P(VDF-TrFE) copolymer strongly absorbs light in the infrared range with wavelength higher than $7 \mu \mathrm{m} .{ }^{[153]}$ This copolymer has been widely used in infrared radiation detectors due to its high absorption of light and good pyroelectric properties. ${ }^{[135,136]}$ Light detection by pyroelectric systems is not limited to utilizing the absorption properties of the pyroelectric material itself, but can also be based on other radiation absorbing methods. Implementing different absorbing electrodes, such as carbon-black, can increase the absorption of incident light and modify the spectral range of light detection. ${ }^{[135,136]}$ In paper I and II, we took advantage of light-induced plasmonic heating in gold nanodisk arrays (paper I) and a perforated gold layer (paper II) to absorb visible light in pyroelectric devices consisting of P(VDF-TRFE) copolymer, which itself is transparent in the visible and near infrared spectral ranges. 


\subsection{Thermal Charging of Supercapacitors}

Supercapacitors are rechargeable energy storage devices with high power densities and long cyclic life, which store charge through one or a combination of two possible mechanisms. ${ }^{[154]}$ Figure 3-7 illustrates a typical electric double-layer capacitor (EDLC) in which the charge storage mechanism involves reversible physical absorption of ions in an electrolyte on the surface of electrodes with high surface area. When a voltage is applied between the two electrodes, positive and negative ions in the electrolyte accumulate at the interface of the electrolyte with the anode and the cathode, respectively. This results in effectively storing electrical energy as surface charges.

Pseudocapacitors use another mechanism to store charges, based on reversible chemical reactions between electrolyte ions and chemically active materials on the electrodes. ${ }^{[154]}$ Another class of supercapacitors called hybrid supercapacitor is merged from the integration of the two aforementioned supercapacitors, which consists of an electric-double layer electrode and a pseudocapacitive electrode.[154, 155]

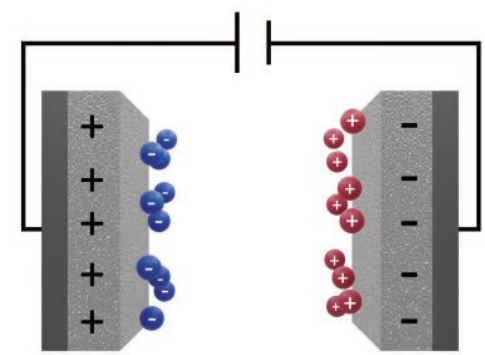

Figure 3-7. schematic illustration of an EDLC capacitor. ${ }^{[154]}$

Supercapacitors have been studied to harvest and store energy from heat through thermally induced charging processes. ${ }^{[133,154,156]} \mathrm{A}$ layer of ions can be spontaneously formed at a metal/electrolyte interface. The excess ions at the interface induce surface charge in the electrode, which affects the electrode potential. A temperature change modifies the electrode potential through, for example, changes in the mobility of the ions and consequently the density of the surface ions. ${ }^{[157]}$ Figure $3-8$ shows a thermally chargeable supercapacitor, which utilizes this process to harvest energy from heat. Each half-cell corresponds to the half of a supercapacitor, consisting of an electrode immersed in an electrolyte solution. ${ }^{[123]}$ Applying a temperature difference between the two half-cells induces an electric potential between the electrodes due to differences in the density of surface ions. In turn, this induced potential difference can drive a current of surface charges from the electrode at higher potential to the one at lower potential. 


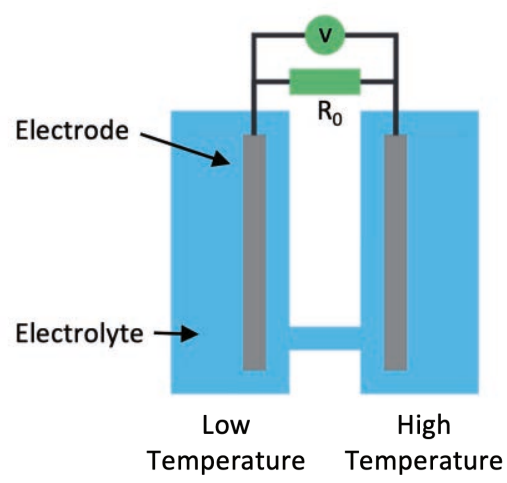

Figure 3-8. Schematic illustration of a thermally-chargeable supercapacitor consisting of two cells maintained at different temperatures and connected through a salt bridge. ${ }^{[158]}$

The conversion of thermal energy to electricity via this capacitive process is independent of thermoelectric or thermogalvanic effects. ${ }^{[123,158]}$

Energy conversion and charge storage properties of the device mostly depend on some factors related to the electrodes and electrolyte, such as the work function and surface area of the electrodes, and the size and mobility of the ions in the electrolyte. ${ }^{[123]}$ Due to the limitations of using liquid electrolyte at high temperatures, such devices can primarily be used to harvest energy from heat sources with low temperatures (less than $200{ }^{\circ} \mathrm{C}$ for devices with ionic-liquid electrolytes). ${ }^{[123]}$

\subsection{Temperature Sensors}

There are many types of temperature sensors, with different temperature operating ranges, accuracy and working principles. Choosing the most suitable sensor depends on the intended application. Temperature sensors can be divided into two categories: contact temperature sensors and non-contact temperature sensors. While the former needs to be in contact with the target object to evaluate the temperature, the latter can detect the temperature remotely based on radiation emitted by the object. ${ }^{[75]}$

A thermostat is a contact temperature sensor or switch, consisting of two different metal bars (such as nickel, copper, aluminum, or tungsten) bonded together to form a bi-metallic strip. The difference in the expansion rates of these metals puts tension on the metal with lower expansion rate leading to a mechanical bending when the system is subjected to a temperature increase. This type of temperature sensor can work as a temperature switch in an electric circuit and is widely used in domestic appliances. ${ }^{[159,160]}$ 
Some other types of contact temperature sensors such as resistance temperature detectors (RTDs) and thermistors monitor the electrical resistance of materials as a function of temperature. ${ }^{[75]}$ In RTDs, metals, such as platinum and constantan (an alloy of $45 \% \mathrm{Ni}$ and $55 \% \mathrm{Cu}$ ), are used as probes which have a stable and almost linear temperature response. ${ }^{[83]}$ A resistance temperature detector with platinum probe has a working temperature range from -100 to $+800{ }^{\circ} \mathrm{C}$ and thermal sensitivity of about $0.39 \Omega \mathrm{K}^{-1} \cdot{ }^{[161]}$ In thermistors, the resistive element is a semiconductor which has a typical exponential temperature response and the temperature range of -100 to +300 ${ }^{\circ} \mathrm{C} .{ }^{75,161]}$ The sensitivity and stability of thermistors are lower than RDTs, however their low cost and wide variety of shapes and sizes have made them popular resistive temperature sensors. ${ }^{[162]}$ Thermistors and RDTs are active resistive devices because they need a power supply to maintain a constant current to pass though the probe, while monitoring the voltage drop. ${ }^{[163]}$ RTDs and thermistors are widely used in various areas such as medical electronics, ${ }^{[164]}$ automotive, ${ }^{[165]}$ computers, and consumer electronics. ${ }^{[166]}$

Thermocouples (TCs) are one of the most inexpensive and most common type of temperature sensors, and their working principle is based on differences in thermoelectric properties of different conductors. ${ }^{[161]}$ As illustrated in Figure 3-9, TCs consist of two dissimilar electrical conductors, such as iron and constantan, which form electrical junctions at the position of the measurement (measuring junction) and at a reference position (reference junction). If the measuring junction and reference junction are at different temperatures, the difference in Seebeck coefficient of the two conductors leads to an electric potential between the junctions, which can be used to evaluate the temperature at the measurement point. There are different types of TCs based on different combinations of conductors, which cover a wide working temperature range from -270 to $+1260{ }^{\circ} \mathrm{C}$ with temperature sensitivity of several tens of $\mu V^{\circ} \mathrm{C}^{-1} \cdot{ }^{[161]}$

Regarding non-contact temperature sensors, pyroelectric detectors discussed in the previous section are widely used for radiation sensing and thermal imaging applications. Thermopiles can also detect thermal radiation via connecting several TCs in series and placing their hot junctions on a thermal radiation absorber and their cold junctions on a heat sink. ${ }^{[167]}$ Radiation thermopiles are used in large spectral range pyranometers to measure the solar irradiance. ${ }^{[168]}$ 


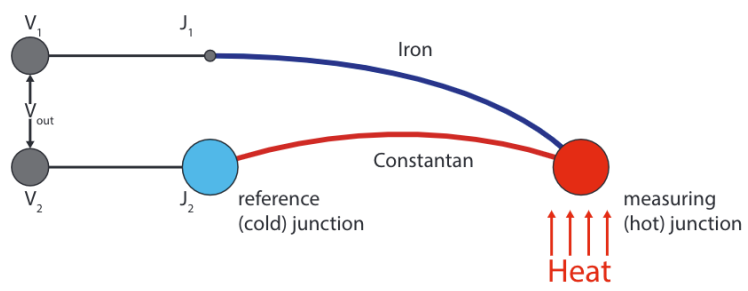

Figure 3-9. Schematic illustration of a thermocouple. ${ }^{[169]}$

Bolometers or calorimeters are another type of non-contact thermal detector that detect the electromagnetic radiation through resistive temperature sensors. There are a wide variety of bolometers such as cooled, uncooled, and composite bolometers that detect infrared and millimeter wavelengths with applications ranging from thermal cameras to astronomical missions. ${ }^{[170]}$

Recently, the concept of self-powered or passive systems has attracted much attention for sensing different stimuli. ${ }^{[11,95,171-176]}$ These sensors eliminate the need for a power supply by generating an electric signal upon an external stimulus. ${ }^{[163]}$ Hence, they can perform all their functions by harvesting energy from the surrounding. ${ }^{95]}$ Among the temperature sensors mentioned above, TCs, radiation thermopiles and pyroelectric detectors are self-powered in the sense that the sensor signals are generated automatically without the need for external power input. These systems have potential applications in technologies such as wearable electronics, humanoid robotics, internet-of-things and medical patches to control health condition of wounds. ${ }^{[91,96,172]}$ 


\section{Chapter 4: Methods and Characterization}

This chapter begins by describing the methods used in this thesis work to deposit organic and inorganic materials on substrates and the techniques employed to produce plasmonic nanohole and nanodisk arrays. Next, the measurement techniques used for evaluating the optical properties of the plasmonic nanostructures are discussed. Finally, the electrical measurement methods employed in this work to evaluate open-circuit voltage, short-circuit current, and the Seebeck coefficient are described.

\subsection{Spin Coating}

Spin coating is a simple and commonly used method to apply uniform thin films of a polymer from solution onto substrates. In this work, this method was employed to obtain thin films of P(VDF-TrFE) copolymer or poly(methyl methacrylate)(PMMA) on different substrates. A substrate is placed on the spinner and a solution containing the polymer is pipetted onto the substrate. Rotation of the spinner (and hence, the substrate) distribute the solution uniformly over the substrate and removes excess solution due to the centrifugal force. The thickness of the final polymer layer depends primarily on the viscosity of the solution and the spin rate. ${ }^{[177]}$

\subsection{Thermal Evaporation}

Thermal evaporation is a physical vapor deposition method for applying metallic thin films on substrates. This method utilizes a vacuum chamber, in which the target metal is placed in a so-called crucible and heated up to its evaporation temperature by passing a high current through the crucible. Under vacuum conditions, vaporized 
atoms of the metal move in a straight lines and deposit on substrates placed upside down some distance away from the crucible, which leads to the creation of a uniform metal layer all over the substrates. Shadow masks can be used during deposition to achieve specific patterns in the metal film. ${ }^{[178]}$

\subsection{Sparse Colloidal Lithography}

A vast amount of research has been dedicated to use various top-down and bottomup techniques to apply plasmonic nanostructures on surfaces. ${ }^{[49]}$ These approaches varies from electron beam lithography and focused ion beam mainly for the fabrication of arbitrary nanostructures, to more scalable methods such as colloidal lithography. ${ }^{[52,53]}$

Self-assembly of colloidal nanoparticles on surfaces can be utilized as a fast and cost-effective method to make large areas of nanostructured surfaces. ${ }^{[61,179]}$ In general, the term colloidal lithography (CL) refers to methods for fabricating such nanostructures using lateral self-organization of colloidal spheres. Sparse colloidal lithography (SCL) is a CL method that was used in this work to fabricate nanohole structures with short-range order on large surfaces. Figure 4-1 shows the process of fabrication of metal nanohole arrays on glass substrates.

Electrostatic interaction between a positively charged substrate and negatively charged polystyrene nanoparticles leads to forming a short-range ordered assembly of the polystyrene nanoparticles on the substrate. ${ }^{[179]}$ Thermal evaporation of a thin metal layer uniformly covers the exposed surface of the polystyrene beads and the interparticle space between them on the glass substrate. Removing the thin-metallayer deposited polystyrene beads with a tape leaves the glass substrate with a uniform metal layer with nanohole arrays screening the position of the polystyrene beads on the surface.

The interparticle distance between the nanoholes can be modified by adding salt (e.g. $\mathrm{NaCl}$ ) to the polystyrene suspension. ${ }^{[61,179,180]}$ The diameter of the nanoholes can be modified by varying the original polystyrene particle size. The thickness of the perforated metal film can be controlled during thermal evaporation of the metal. In paper II and IV, $30 \mathrm{~nm}$-thick gold nanohole arrays with diameters of $160 \mathrm{~nm}$ and characteristic interparticle distance of $325 \mathrm{~nm}$ thickness of $30 \mathrm{~nm}$ were fabricated.

In Paper III, nanohole arrays of gold and aluminum with diameter of $107 \mathrm{~nm}$ and thickness of $20 \mathrm{~nm}$ were obtained by the SCL method. For samples with gold nanohole arrays, a $3 \mathrm{~nm}$-thick titanium was evaporated before depositing gold layer to increase the adhesion of gold to glass substrate. 


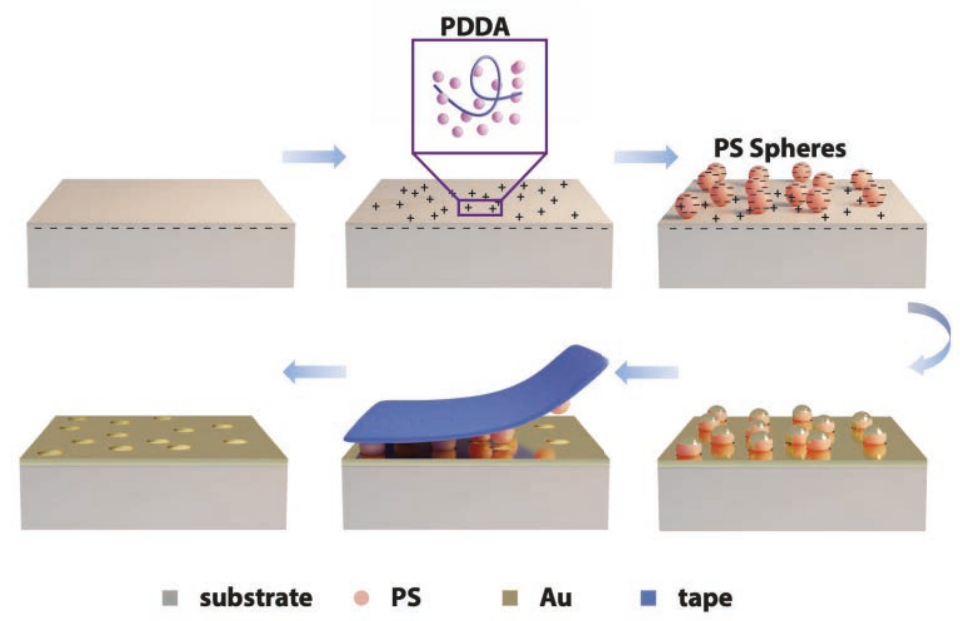

Figure 4-1. Schematic illustration of different steps for fabricating gold nanohole arrays on a glass substrate. ${ }^{[180]}$

\subsection{Hole-mask Colloidal Lithography}

Hole-mask colloidal lithography (HCL) can be considered as an extension of the CL method. This method was employed in paper I to fabricate uniform distribution of short-range ordered gold nanodisk arrays on glass ITO substrates (Figure 4-2). Compared to the CL method, HCL includes two new essential features which are a sacrificial layer and a thin metallic nanohole mask. ${ }^{[179]}$ Before depositing polystyrene beads, it is necessary to apply a sacrificial layer such as PMMA layer on the substrate. Through CL method, a metallic mask with nanoholes is deposited on the sacrificial layer. Oxygen plasma treatment allows transferring the hole-mask pattern into the sacrificial layer and remains a thin film mask supported by perforated sacrificial layer (PMMA) on the substrate surface. Deposition of another metal layer and subsequent lift-off of the sacrificial layer result in fabrication of a short-range ordered metallic nanodisk arrays on the substrate. ${ }^{[179]}$

Similar to SCL, the dimeter of nanodisks and the characteristic particle distance between them can be controlled by changing the original polystyrene particle size and adding salt to polyelectrolyte solution, respectively. 


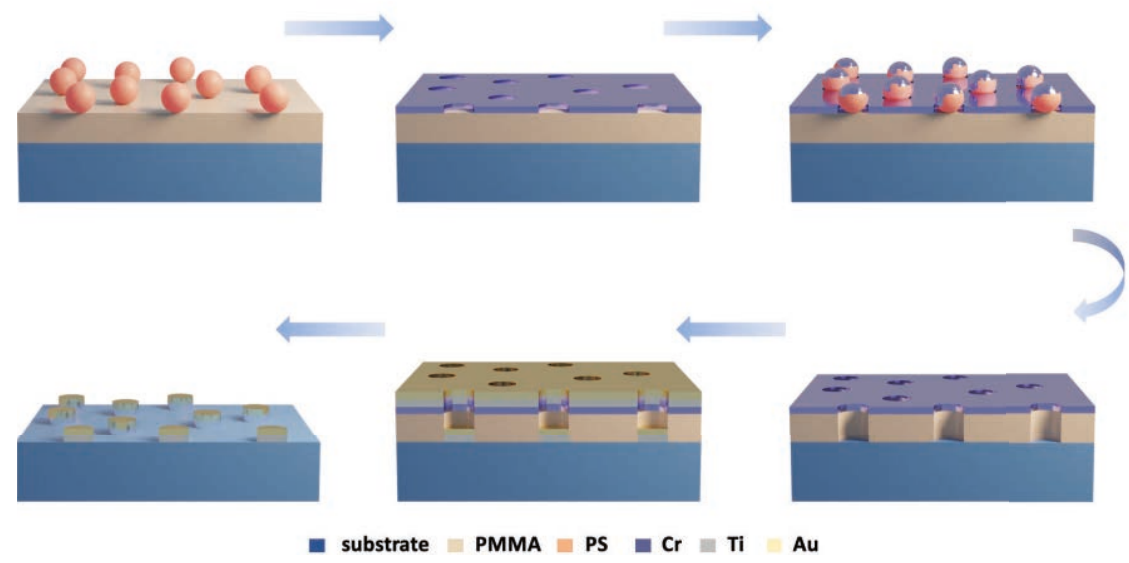

Figure 4-2. Fabrication process of gold nanodisk arrays using hole-mask colloidal lithography.

\subsection{Extinction Spectroscopy}

As discussed in Chapter 2, interaction of light with plasmonic nanostructures typically results in high resonant absorption $(A)$ and scattering $(S)$ and consequently a corresponding reduction in the transmitted light around the resonance frequency. In extinction spectroscopy, absorption and scattering are measured together as the amount of light not transmitted through the sample. ${ }^{[181]}$

Extinction $(E)$ is determined by measuring the ratio of transmitted $(I)$ to incident $\left(I_{0}\right)$ irradiance, and has values from 0 (no extinction) to 1 (no transmission): ${ }^{[46]}$

$$
E=1-\frac{I}{I_{0}} \quad(4-1)
$$

where the ratio $I / I_{0}$ is the transmittance $(T)$. Figure 4-3 depicts the extinction measurement setup used in paper I. In this setup, light from a white light source (300 W, Xe lamp, Newport) is collimated using a lens and transmitted through the sample and detected using an optical light guide attached to a spectrograph (Andor Shamrock 303i, Newton CCD detector). 


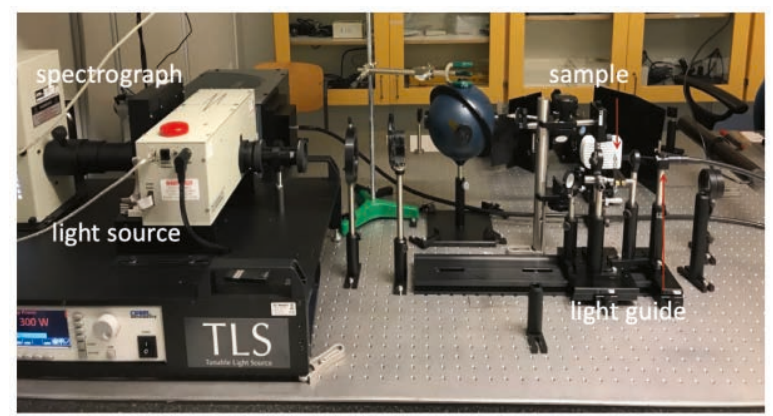

Figure 4-3. Photograph of the measurement setup used in this work to measure the extinction of nanodisk samples.

\subsection{Absorption Spectroscopy}

Since the extinction is the sum of absorption and scattering $(E=A+S){ }^{[46]}$ only using extinction spectroscopy is not enough to determine the relative contribution of absorption and scattering to extinction. To determine the individual contributions, an additional independent observation is needed, such as measuring the absorption with an integrating sphere. An integrating sphere is a hollow spherical cavity for measuring optical radiation. ${ }^{[56]}$ The interior of the sphere is covered with a diffuse reflective coating. There are some openings in the sphere for light to enter, to insert the sample and exit ports to collect light and send it to a detector such as a spectrograph. For the absorption measurements in paper II, III, and IV, samples were placed in the middle of the integrating sphere (Figure 4-4a). In this manner, light transmitted through the sample and light reflected or scattered by the sample were both collected and transferred to the spectrograph using the optical guide (Figure 4-4b).

a)

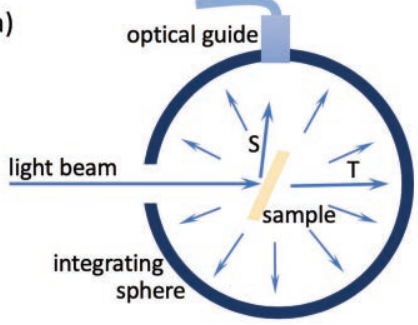

b)

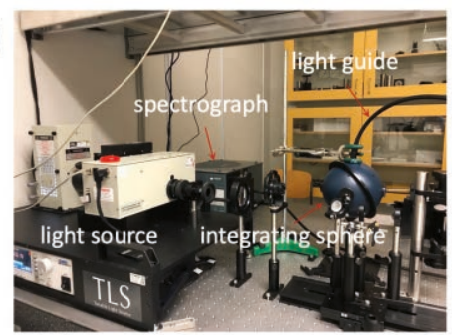

Figure 4-4. a) Schematic illustration of a scattering/transmission measurement in the integrating sphere to evaluate the absorption of the nanohole arrays. b) Photograph of the measurement setup used in this thesis work, which includes integrating sphere. 
The absorption could then be obtained directly from this measurement as: ${ }^{[46,56]}$

$$
\begin{array}{ll}
E=A+S=1-T, & (4-2) \\
A=1-(S+T) & (4-3)
\end{array}
$$

where the values for absorption varies from 0 (no absorption) to 1 (no total scattering).

\subsection{Radiation-induced Voltage Measurements}

To measure the voltage generated by different devices upon irradiation (in paper I, II, III and IV), we used a solar simulator (LCS-100, Oriel Instruments, $300 \mathrm{~W}$ Xe lamp, AM1.5G filter) calibrated to an irradiance of 1 Sun $\left(1000 \mathrm{~W} \mathrm{~m}^{-2}\right)$ as the source of light and a Keithley 2182A nano-voltmeter for measuring the generated voltage. In Paper I, the voltage over an external load ( $9 \mathrm{M} \Omega$ resistor) was measured and a manual shutter was employed to block the light over the hybrid device, except for two measurements where a leaf of a plant was used instead of the shutter. The power generated by the device was calculated using:

$$
W=\frac{V^{2}}{R} \quad(4-4)
$$

where $V$ is the measured voltage and $R$ is the resistance of the load $(9 \mathrm{M} \Omega)$ in the circuit.

In paper II, III and IV, we measured open-circuit voltages generated by the devices upon irradiation. Light on and off states were controlled using an optical beam shutter (SH, Thorlabs).

\subsection{Direct Heating Voltage Measurements}

In paper II, III and IV, open-circuit voltages generated by the devices were measured using a Keithley 2182A nano-voltmeter during controlled temperature cycles enabled by placing the samples on a LabVIEW-controlled Peltier element.

\subsection{Pyroelectric Current Measurement}

In paper I, short-circuit current generated by the devices upon light fluctuations was measured using a Keithley 4200-SCS parameter analyzer provided with two highresolution SMUs. For this measurement, a solar simulator (LCS-100, Oriel Instruments, $300 \mathrm{~W}$ Xe lamp, AM1.5G filter) calibrated to an irradiance of 1 Sun (1000 $\mathrm{W} \mathrm{\textrm {m } ^ { - 2 }}$ ) was used as the source of light, while the light on and off states were controlled manually. 


\subsection{Seebeck Coefficient Measurement}

A lateral device as depicted in Figure 4-5 was used to measure the Seebeck coefficient of the polyelectrolytes used in paper II and III. The samples where dropcasted onto a glass substrate with two gold electrodes (deposited via shadow mask evaporation) and placed on two Peltier elements to apply controlled heat cycles. A nanovoltmeter monitored the open-circuit voltage $(\Delta V)$ while the temperature difference $(\Delta T)$ between the two electrodes was measured using two TCs. The Seebeck coefficient was calculated from the slop of a linear fit of $\Delta V$ versus $\Delta T$.

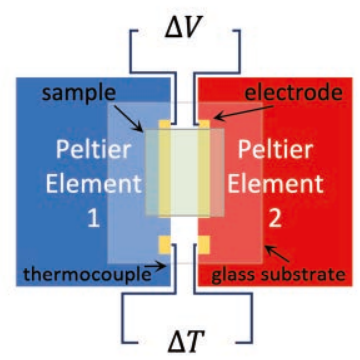

Figure 4-5. Schematic illustration of the Seebeck coefficient measurement setup. ${ }^{[132]}$ 


\section{Chapter 5: Summary of the appended papers}

\footnotetext{
5.1. Harvesting Energy from Light Fluctuations - Paper I

In paper I, fast and efficient plasmonic heating in combination with the pyroelectric effect allow a plasmonic-pyroelectric (plasmo-pyro) hybrid device to harvest energy from light fluctuations. The device consisted of an array of gold nanodisks deposited by hole-mask colloidal lithography on a glass ITO substrate, a spin-coated layer of P(VDF-TrFE) copolymer, and a gold top electrode deposited by shadow mask evaporation. Plasmonic heating in the nanodisks leads to increasing the temperature of the nanostructure and its surrounding media upon light illumination. The plasmo-pyro hybrid device could translate the temperature variations to an electric output signal through changing the polarization strength of $\mathrm{P}(\mathrm{VDF}-\mathrm{TrFE})$ because of the thermal movement of the molecules in the copolymer layer. Later in the absence of light, the plasmo-pyro hybrid device generated another transient pyroelectric current with opposite polarity.

The polarization status of the $\mathrm{P}(\mathrm{VDF}-\mathrm{TrFE})$ copolymer is found as a vital factor influencing the response of the plasmo-pyro hybrid device. As discussed in Chapter 3, a $\mathrm{P}(\mathrm{VDF}-\mathrm{TrFE})$ film has zero residual dipole moment (non-polarized) after spin coating, because of the random orientation of the dipoles. The light blue line in Figure 5-1 indicates the response of the non-polarized hybrid device upon temperature changes originated from the plasmonic heating.
} 


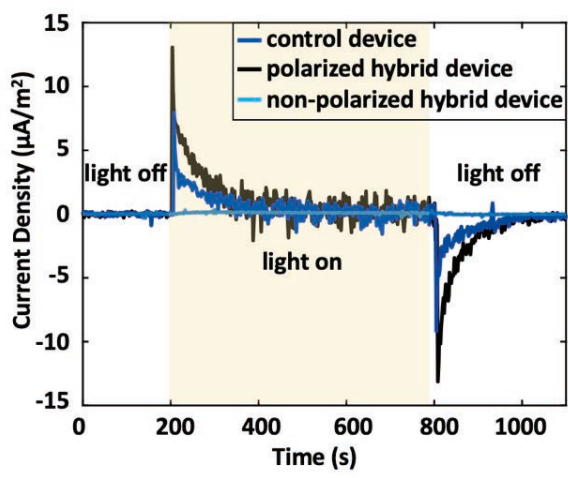

Figure 5-1. Short-circuit current density generated by a polarized plasmo-pyro hybrid device (black line), a control device (dark blue line), and a non-polarized plasmo-pyro hybrid device (light blue line) upon fluctuating simulated solar illumination (1 Sun, light on/off). Adapted with permission from reference [149].

The lack of residual dipole moment in the non-polarized hybrid device disables it from converting plasmonic heating into electric signals. However, the dipoles could be aligned with an applied electric field through pooling process. This gave a permanent residual dipole moment across the $\mathrm{P}(\mathrm{VDF}-\mathrm{TrFE})$ film and resulted in a polarized plasmo-pyro hybrid device which could convert the light-induced temperature fluctuations to electric signals (black line in Figure 5-1).

The plasmonic heating in the gold nanodisk arrays is another influential factor on the current density generated by the plasmo-pyro hybrid device. Figure 5-1 compares the short-circuit current density of such hybrid device (black line in Figure 5-1) with that of a control device (dark blue line in Figure 5-1) consisting similar structure as the plasmo-pyro hybrid device, but without gold nanodisk arrays. These results show that the plasmo-pyro hybrid device could produce higher current density due to the fast and efficient plasmonic heating in the gold nanodisk arrays under light illumination.

Fluctuations of solar irradiance due to natural processes, such as tree branches swinging in the wind or clouds moving over the sky can negatively affect the efficiency of conventional photovoltaic solar cells, whereas it is a necessary condition for our plasmo-pyro hybrid device to generate power. Figure $5-2 \mathrm{~b}$ shows that the polarized plasmo-pyro hybrid device could generate electric power in an external circuit upon illumination of light from a solar simulator which was randomly interrupted by a leaf swinging in the wind above the device (Figure 5-2a). 
a)

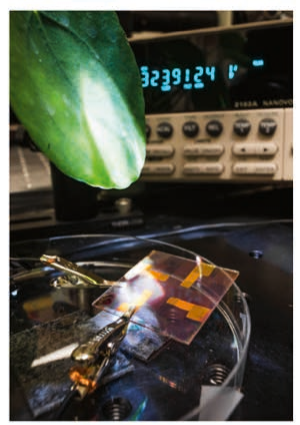

b)

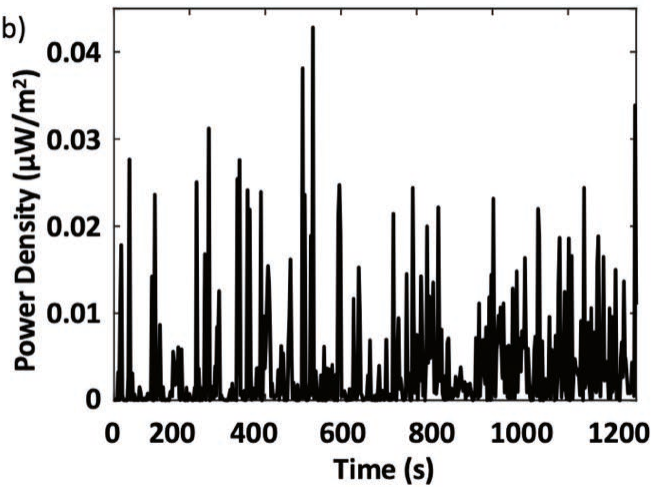

Figure 5-2. a) The image of the experimental setup for random light fluctuation upon plasmo-pyro hybrid device (Image by Thor Balkhed). b) The power density generated by the polarized plasmo-pyro hybrid device (black line) upon random light fluctuation using a swinging leaf blocking light from solar simulator (1 sun illumination/shade). Adapted with permission from reference [149].

Harvesting energy from light using plasmonic nanostructures in inorganic hybrid systems have been studied earlier. There are some reports on utilizing high optical scattering properties of metallic nanoparticles in photovoltaic solar cells that enhances the absorption of light through light trapping and consequently improves the efficiency of photovoltaic solar cells. ${ }^{[182-184]}$

In another work by Xiong Y, et al., the high absorption of light in metallic nanoparticles and corresponding heat generation induces a temperature gradient over an inorganic thermoelectric generator which enables the generation of a thermoelectric power. ${ }^{[13]}$ Similar to our hybrid device, these systems benefit from plasmonic properties of metallic nanostructures, but the efficiency in these systems tends to reduce under fluctuation of light.

Pyroelectric materials have piezoelectric properties as well. In a subsequent work, the light-induced plasmonic heating and piezoelectric interaction for energy harvesting applications were investigated by Ghim Wei Ho group. ${ }^{[185]}$ They reported a piezo-response enhancement in a PVDF film because of the thermal-expansioninduced local strain of the polymer originated from the plasmonic photothermal effect of silver nanoparticles embedded in the PVDF. ${ }^{[185]}$ 


\subsection{Three Effects in One Concept for Sensing Radiation and Heat - Paper II}

Ionic thermoelectric materials are suitable for sensing temperature in e-skin applications thanks to their high temperature sensitivity and good mechanical flexibility. However, they are slow in sensing temperature variations, due to low mobility and slow thermodiffusion of the ions. In contrast, temperature sensors consisting of pyroelectric are quick in tracing

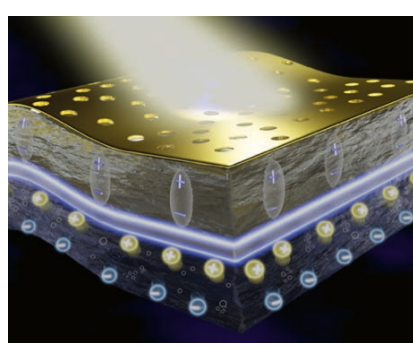
variations in temperature by generating transient signals, but incapable of detecting equilibrium temperatures. Paper II introduces a hybrid device called integrated pyroelectric-ionic thermoelectric (pyro-iTE) device to address these deficiencies. The concept combined an ionic thermoelectric material with a pyroelectric capacitor. More precisely, the integrated pyro-iTE device consisted of a thin pyroelectric layer (P(VDF-TrFE)) sandwiched between two electrodes, and an ionic thermoelectric gel ([EMIM][TFSI]) was placed on top of the upper electrode. Results from open-circuit voltage measurement in Figure 5-3b show that this system provides both quick initial (pyroelectric) response and stable (thermoelectric) signals (orange line) upon prolonged heating (temperature cycles are shown in Figure 5-3a). The stable signals at equilibrium generated by the integrated pyro-iTE device were enhanced compared with signals expected from the Seebeck coefficient of the ionic thermoelectric material (red line in Figure 5-3b).

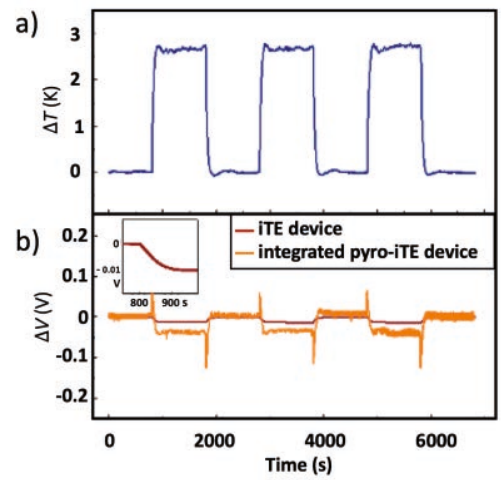

Figure 5-3. a) Temperature cycles applied to the ionic thermoelectric and integrated pyroiTE device. b) Open-circuit voltage generated by the ionic thermoelectric material (red line) and integrated pyro-iTE device (orange line). The inset shows the magnification view of the

first signal that the ionic thermoelectric material generated upon the first temperature cycle. Adapted with permission from reference [132]. 
This enhancement of the signals at equilibrium is primarily attributed to the interactions between ions in the electrolyte and the high density of charges at the $\mathrm{P}(\mathrm{VDF}-\mathrm{TrFE}) /$ electrode interfaces.Moreover, continuous bottom electrode of the pyroelectric compartment of the integrated pyro-iTE device was replaced by a gold nanohole layer. Plasmonic heating in such metasurface electrode enabled the concept to efficiently detect heat from radiation upon solar light illumination. The hybrid concept can sense heat from a warm touch, radiation, and direct changes in temperature of the environment. These promising properties may find use in applications such as e-skin and humanoid robotics.

\subsection{Simple Concepts with Outstanding Temperature Sensing Properties - Papers III and IV}

TCs are suitable temperature sensors for skin-inspired wearable applications owing to the ease of use and autonomous sensing properties. ${ }^{[186]}$ However, the sensitivity of TCs is limited to a few tens of microvolt per kelvin, which might be insufficient for applications such as health monitoring and soft robotics that require temperature sensing with higher sensitivity as well as ease of signal detection. ${ }^{\text {92, }}$ 95]

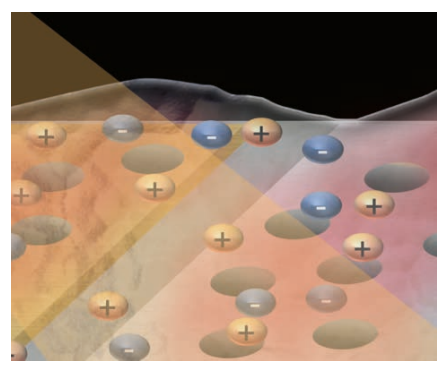
Paper III presents a concept called electrolyte-assisted temperature sensor (EATS) which analogously to a traditional TC can autonomously generate signals upon temperature changes but with about 300 times higher magnitudes. As shown in Figure 5-4, the EATS has orders of magnitude higher sensitivity compared to that of a traditional TC made up of aluminum and gold metal electrodes and that of commercial TCs.

Studies on the mechanism of the device in sensing temperature indicated that the generation of the large and autonomous signals is independent of a specific temperature gradient, and that it is related to the variations in the temperature of the redox-active aluminum electrode while being connected through the electrolyte to the inert gold electrode. The EATS is also sensitive to humidity since it affects the total resistance of the sensor. 


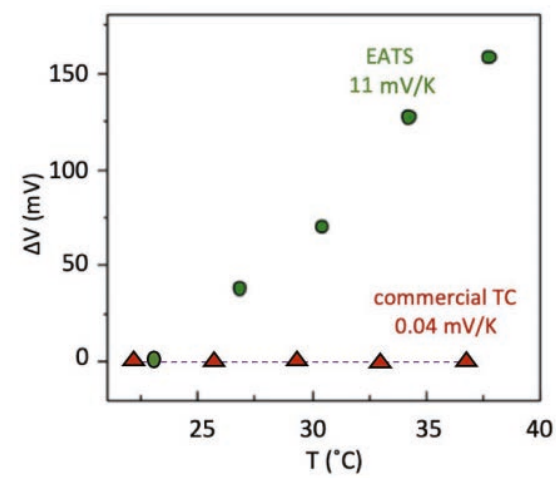

Figure 5-4. Open-circuit voltage vs temperature plot of EATS (green dots) and a commercial thermocouple (red rectangles).

For some applications, such as e-skin, it is important to decouple the signals. We showed that, encapsulating the devices enabled EATS to monitor temperature without an influence from other stimuli, especially humidity variations. Moreover, the encapsulated temperature sensor has a higher sensitivity to a human's finger touch than a commercial TC, regardless of the humidity level of the surroundings. The simple structure of the EATS together with its promising sensing properties make the concept suitable for applications requiring temperature mapping.

a)

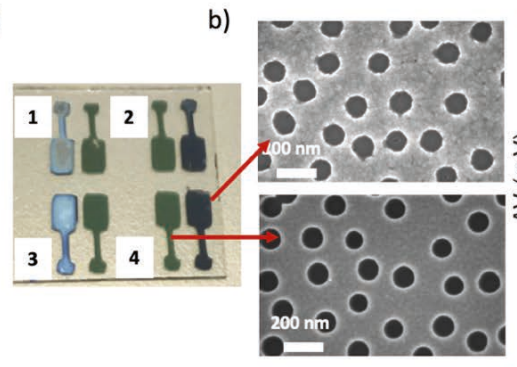

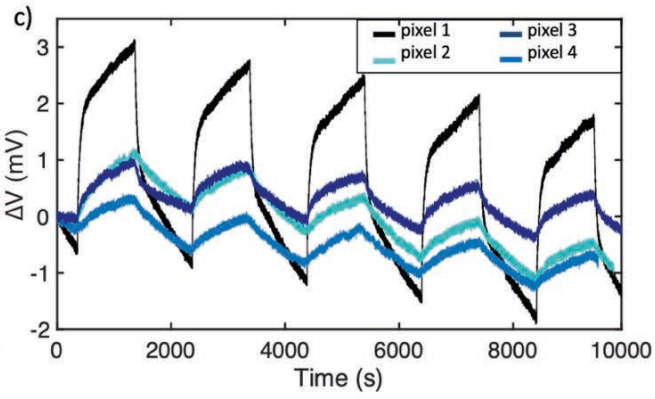

Figure 5-5. a) Photograph of four electrolyte-assisted temperature sensors consisting of perforated gold and aluminum electrodes on a glass substrate. b) SEM images of aluminum

(upper image) and gold (bottom image) nanohole electrodes. c) Open-circuit voltage generated by pixel 1 (black line), 2 (canyon line), 3 (dark blue line), and 4 (light blue line) shown in the image of the arrays in (a) while illuminating pixel 1 with visible light from a solar simulator (1 sun). 


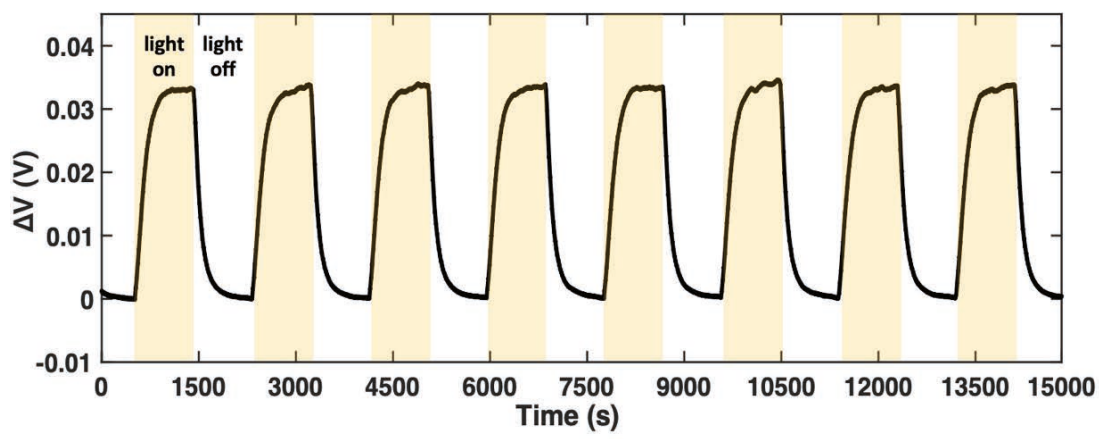

Figure 5-6. Open-circuit voltage generated by vertical temperature sensor with perforated gold electrode upon solar simulator illumination (1 sun).

An array consisting of four electrolyte-assisted temperature sensors on a substrate (Figure 5-5a) allows the mapping of an applied temperature profile. Augmentation of the EATS sensing properties to detect heat from radiation may be favorable for e-skin and other applications. Replacing the continuous metal electrodes with plasmonic aluminum (Figure 5-5b upper panel) and gold (Figure 5-5b bottom panel) nanohole electrodes increases the sensitivity of the devices to visible light (Figure 5-5c) through plasmonic heating of the illuminated metasurface electrodes.

It is crucial that the temperature sensitivity of the individual temperature sensors and temperature mapping resolution are optimized to achieve a temperature mapping matrix with suitable mapping properties. The main aim in paper IV was to increase the resolution of the temperature mapping of the sensor matrix presented in paper III by modifying the structure of the temperature sensors from lateral form to vertical form. In such configuration, the ionic liquid-based electrolyte was sandwiched inside a cavity between the two dissimilar electrodes (aluminum and gold). The temperature sensitivity of the vertical temperature sensors is similar to that of the EATS. A $3 \times 3$ matrix of such temperature sensors was capable of mapping temperature autonomously with better resolution compared to the EATS. Replacing the continuous gold electrode with perforated gold layer in the temperature sensor allowed it to detect the heat from visible light radiation (Figure 5-6) more effectively. Similar to the other hybrid plasmonic sensors presented in this thesis work, such hybrid plasmonic sensor could detect variation in temperature through both radiation-induced heating and direct heating. 


\section{Chapter 6: Conclusion and Outlook}

The emphasis of this thesis work has been on developing hybrid plasmonic systems for energy harvesting and heat and radiation sensing applications. In particular, we have utilized the strong light absorption of plasmonic metal nanostructures for photo-thermal conversion processes, and combined such optical heating with other functional materials.

To harvest energy from light fluctuations, we designed a system made up of gold nanodisk arrays and pyroelectric $\mathrm{P}(\mathrm{VDF}-\mathrm{TrFE})$ copolymer (presented in Paper I) that takes advantage of the fast light-induced plasmonic heating in the plasmonic nanostructure and the pyroelectric conversion in the copolymer. This system is an example of a hybrid plasmonic system that can deliver a property that is achievable only if the components are combined together into one system. The short rangeordered metal nanodisk arrays have a relatively narrow plasmonic resonance band and their resonance wavelength can be tuned in the visible to near infrared wavelength ranges by varying the geometry of the nanodisks. ${ }^{[53]}$ Owing to this tunability, such hybrid plasmo-pyroelectric devices could be designed to generate voltages in response to specific ranges of wavelengths. Indeed, a recent study by Stewart et al. demonstrated ultrafast inorganic pyroelectric photodetection using plasmonic spectral filters. ${ }^{[187]}$ Placing different wavelength responsive plasmo-pyro hybrid devices together as an array could, for example, be used as spectrally sensitive light sensors.

As example of other possible future application of plasmo-pyro hybrid devices, they could be used as energy harvesting windows, by fabricating nanodisk arrays of inexpensive and abundant metals such as aluminum. Similar to gold nanodisk arrays, the plasmon resonance of the aluminum nanodisks can be tuned to absorb light in the 
near-infrared range and transmit the visible light, through choosing proper dimensions and inter particle distance. ${ }^{[15]}$ I believe such concept can potentially also harvest energy from mechanical vibrations caused by rain drops hitting the window, since the $\mathrm{P}(\mathrm{VDF}-\mathrm{TrFE})$ layer has piezoelectric properties as well.

Through the work of this thesis we have developed and studied several hybrid plasmonic systems for detection of direct heating and light-induced heating (Papers II-IV). These studies utilized gold nanohole arrays for plasmonic photothermal conversion while they also served as electrodes to collect electric signals. In paper II, we combined a pyroelectric and a thermoelectric material with gold nanohole arrays to detect heat from visible light radiation as well as from direct contact with a warm object. The merits of such system (i.e. the combination of pyroelectrics and ionic thermoelectrics) over its individual components was that it could rapidly detect heat stimuli and also generate enhanced stable signals upon continuous heating. Such concept may have some applications in e-skin to provide humanoid robotics and prosthetic limbs with sensation of radiation and touch of warm objects. E-skin can also be used for monitoring different parameters on human skin. In that case it performs as a "second skin" in humans, so it should preferably hold some similar mechanical properties as human skin has. ${ }^{[188]}$ As a future outlook, to fulfil such requirement, the hybrid device could be incorporated into flexible arrays. Moreover, despite our efforts to explain the mechanism of signal generation in such hybrid device, further studies are needed to gain better understanding of the interactions between ions and surface charges and local electric potential, by using methods such as Kelvin probe force microscopy. Such comprehensive understanding will probably help to further enhance the hybrid device performance.

We also showed that temperature sensors with high sensitivity can be constructed with rather simple structures. This temperature sensor is a hybrid plasmonic system made of nano-perforated thin metal film combined with a gel-like electrolyte (Paper III) and it could generate high open-circuit voltage upon heat and radiation stimuli. In terms of future applications, it is worth noting that the electrolyte that was used in those devices is compatible with printing techniques. This can allow for fast and inexpensive production of such temperature sensors, for example, for medical care applications. As continuation of this thesis work, it would be interesting to study the sensing properties and performance of hybrid sensors produced though such printing techniques. We showed that a device consisting of an array of such sensors could map heat distribution generated by direct heating or radiation stimuli. In paper IV, we improved the conditions for temperature mapping by modifying the device from lateral to vertical structure. Good temperature sensing and mapping properties 
together with simple structure and low production costs could make the concept interesting for many applications requiring contact and/or non-contact temperature sensors, such as in smart buildings to monitor and control the temperature, in smart agriculture industry to supervise the irrigation of farm fields at proper time based on the weather conditions, in electrical devices such as mobile phones and monitors to automatically adjust the brightness of the screen, or to work as receivers for remote control devices. Further investigations may study effects of the Seebeck coefficient of the gel-like electrolyte on the temperature sensitivity of these types of sensors. This can be done by modifying the thickness of the spacer between the two electrodes of the vertical temperature sensor and measuring the open-circuit voltage generated by the sensors upon applying controlled temperature cycles. These investigations may help to understand the mechanism of signal generation and optimizing the performance of the sensor.

As an outlook for utilizing plasmonic heating in hybrid systems, one could consider many other applications, such as light-responsive devices based on combining thermoplasmonic surfaces with polymers that change volume upon temperature changes (such as poly(N-isopropylacrylamide) (PNIPAM)). ${ }^{[189,}$ 190] Such hybrid thermoplasmonic systems could enable systems with optical properties that change (or adapt) to illumination conditions.

In summary, the work presented in this thesis introduced novel applications and perspective for the plasmonic heating effect through integrating metal nanostructures with organic functional materials. Those were some of the numerous directions and possibilities to benefit from plasmonic heating in hybrid systems, and there are many interesting future studies to undertake. 


\section{References}

[1] G. Sun and J. B. Khurgin, "Plasmonics and Plasmonic Metamaterials," in World Scientific Series in Nanoscience and Nanotechnology, vol. 4: World Scientific, 2011, pp. 1-44.

[2] J. Z. Zhang and C. Noguez, "Plasmonic Optical Properties and Applications of Metal Nanostructures," Plasmonics, vol. 3, no. 4, pp. 127-150, 2008.

[3] W. Q. Lim and Z. Gao, "Plasmonic Nanoparticles in Biomedicine," Nano Today, vol. 11, no. 2, pp. 168-188, 2016.

[4] S. V. Boriskina, H. Ghasemi, and G. Chen, "Plasmonic Materials for Energy: From Physics to Applications," Materials Today, vol. 16, no. 10, pp. 375-386, 2013.

[5] M. Pelton, J. Aizpurua, and G. Bryant, "Metal-Nanoparticle Plasmonics," Laser and Photonics Reviews, vol. 2, no. 3, pp. 136-159, 2008.

[6] V. Amendola, R. Pilot, M. Frasconi, O. M. Maragò, and M. A. Iatì, "Surface Plasmon Resonance in Gold Nanoparticles: A Review," Journal of Physics: Condensed Matter, vol. 29, no. 20, pp. 203002-203002, 2017.

[7] W. Fan and M. K. H. Leung, "Recent Development of Plasmonic Resonance-Based Photocatalysis and Photovoltaics for Solar Utilization," Molecules vol. 21, no. 2, pp. 180-206, 2016.

[8] G. E. Jonsson, V. Miljkovic, and A. Dmitriev, "Nanoplasmon-Enabled Macroscopic Thermal Management," Scientific Reports, vol. 4, pp. 1-7, 2014.

[9] G. Baffou and R. Quidant, "Thermo-Plasmonics: Using Metallic Nanostructures as Nano-Sources of Heat," Laser \& Photonics Reviews, vol. 7, no. 2, pp. 171-187, 2013.

[10] L. Jauffred, A. Samadi, H. Klingberg, P. M. Bendix, and L. B. Oddershede, "Plasmonic Heating of Nanostructures," Chemical Reviews, pp. 8087-8130, 2019.

[11] H. Yu, Y. Peng, Y. Yang, and Z.-Y. Li, "Plasmon-Enhanced Light-Matter Interactions and Applications," npj Computational Materials, vol. 5, no. 1, p. 45, 2019.

[12] J. S. Donner, G. Baffou, D. McCloskey, and R. Quidant, "Plasmon-Assisted Optofluidics," ACS Nano, vol. 5, no. 7, pp. 5457-5462, 2011.

[13] Y. Xiong et al., "Solar Energy Conversion with Tunable Plasmonic Nanostructures for Thermoelectric Devices," Nanoscale, vol. 4, no. 15, pp. 4416-4420, 2012.

[14] P. Campbell, "Light Trapping in Textured Solar Cells," Solar Energy Materials, vol. 21, no. 2, pp. 165-172, 1990.

[15] I. Zorić, M. Zäch, B. Kasemo, and C. Langhammer, "Gold, Platinum, and Aluminum Nanodisk Plasmons: Material Independence, Subradiance, and Damping Mechanisms," ACS Nano, vol. 5, no. 4, pp. 2535-2546, 2011.

[16] P. K. Jain, X. Huang, I. H. El-Sayed, and M. A. El-Sayed, "Noble Metals on the Nanoscale: Optical and Photothermal Properties and Some Applications in Imaging, Sensing, Biology, and Medicine," Accounts of Chemical Research, vol. 41, no. 12, pp. 1578-1586, 2008.

[17] M. A. El-Sayed and S. Link, "Shape and Size Dependence of Radiative, Non-Radiative and Photothermal Properties of Gold Nanocrystals," International Reviews in Physical Chemistry, vol. 19, no. 3, pp. 409-453, 2000.

[18] C. Liow, F. Meng, X. Chen, and S. Li, "Dependence of Plasmonic Properties on Electron Densities for Various Coupled Au Nanostructures," Journal of Physical Chemistry C, vol. 118, no. 47, pp. 27531-27538, 2014. 
[19] M. T. Sheldon, J. V. De Groep, A. M. Brown, A. Polman, and H. A. Atwater, "Plasmoelectric Potentials in Metal Nanostructures," Science, vol. 346, no. 6211, pp. 828-831, 2014.

[20] J. Zhang, L. Zhang, and W. Xu, "Surface Plasmon Polaritons: Physics and Applications," Journal of Physics D: Applied Physics, vol. 45, no. 11, 2012.

[21] K. Zhelyazkova, M. Petrov, B. Katranchev, and G. Dyankov, "Surface Plasmon Resonance on the Surface: Metal - Liquid Crystal Layer," Journal of Physics: Conference Series, vol. 558, no. 1, 2014.

[22] T. W. Ebbesen, H. J. Lezec, H. F. Ghaemi, T. Thio, and P. A. Wolff, "Extraordinary Optical Transmission through Sub-Wavelength Hole Arrays," Nature, vol. 391, no. 6668, pp. 667-669, 1998.

[23] V. Klimov, "Surface Plasmons," in Nanoplasmonics: Pan Stanford, 2014, pp. 63-90.

[24] S. A. Maier and H. A. Atwater, "Plasmonics: Localization and Guiding of Electromagnetic Energy in Metal/Dielectric Structures," Journal of Applied Physics, vol. 98, no. 1, p. 011101, 2005.

[25] P. Nordlander, T. H. Park, N. Mirin, J. B. Lassiter, C. L. Nehl, and N. J. Halas, "Optical Properties of a Nanosized Hole in a Thin Metallic Film," Acs Nano, vol. 2, no. 1, pp. 25-32, 2008.

[26] E. Martinsson, "Nanoplasmonic Sensing Using Metal Nanoparticles," PhD dissertation, Department of Physics, Chemistry and Biology Linköping University, Linköping University Electronic Press, 2014.

[27] Kojaro. (2019). Nasirol Molk Mosque. Available: https://www.kojaro.com

[28] C. Li, C. Wang, Q. Han, Y. Wu, and H. Zheng, "Investigation on Optical Properties of Ag-Au Alloy Nanoparticles," Plasmonics, vol. 12, no. 5, pp. 1373-1379, 2017.

[29] I. Freestone, N. Meeks, M. Sax, and C. Higgitt, "The Lycurgus Cup - a Roman Nanotechnology," Gold Bulletin, vol. 40, no. 4, pp. 270-277, 2007.

[30] X. I. Chen, "Photothermal Effect in Plasmonic Nanostructures and Its Applications," $\mathrm{PhD}$ dissertation, KTH School of Information and Communication Technology, Kungl Tekniska högskolan, 2014.

[31] G. Baffou and R. Quidant, "Thermo-Plasmonics: Using Metallic Nanostructures as Nano-Sources of Heat," Laser and Photonics Reviews, vol. 7, no. 2, pp. 171-187, 2013.

[32] S. A. Maier, Plasmonics: Fundamentals and Applications. New York: Springer, 2007.

[33] A. V. Zayats and I. I. Smolyaninov, "Near-Field Photonics: Surface Plasmon Polaritons and Localized Surface Plasmons," Journal of Optics A: Pure and Applied Optics, vol. 5, no. 4, p. S16, 2003.

[34] W. L. Barnes, A. Dereux, and T. W. Ebbesen, "Surface Plasmon Subwavelength Optics," Nature, vol. 424, no. 6950, pp. 824-830, 2003.

[35] H. L. Chen, K. C. Hsieh, C. H. Lin, and S. H. Chen, "Using Direct Nanoimprinting of Ferroelectric Films to Prepare Devices Exhibiting Bi-Directionally Tunable Surface Plasmon Resonances," Nanotechnology, vol. 19, no. 43, p. 435304, 2008.

[36] H. Frederich et al., "Determination of the Surface Plasmon Polariton Extraction Efficiency from a Self-Assembled Plasmonic Crystal," Plasmonics, vol. 9, no. 4, pp. 917-924, 2014.

[37] J. M. Pitarke, V. M. Silkin, E. V. Chulkov, and P. M. Echenique, "Theory of Surface Plasmons and Surface-Plasmon Polaritons," Reports on Progress in Physics, vol. 70, no. 1, pp. 1-87, 2006.

[38] K. A. Willets and R. P. Van Duyne, "Localized Surface Plasmon Resonance Spectroscopy and Sensing," Annual Review of Physical Chemistry, vol. 58, no. 1, pp. 267-297, 2007. 
[39] E. Petryayeva and U. J. Krull, "Localized Surface Plasmon Resonance: Nanostructures, Bioassays and Biosensing - a Review," Analytica Chimica Acta, vol. 706, no. 1, pp. 824, 2011.

[40] M. A. El-Sayed and S. Link, "Shape and Size Dependence of Radiative, Non-Radiative and Photothermal Properties of Gold Nanocrystals," International Reviews in Physical Chemistry, vol. 19, no. 3, pp. 409-409, 2000.

[41] P. Colomban, "The Use of Metal Nanoparticles to Produce Yellow, Red and Iridescent Colour, from Bronze Age to Present Times in Lustre Pottery and Glass: Solid State Chemistry, Spectroscopy and Nanostructure," Journal of Nano Research, vol. 8, pp. 109-132, 2009.

[42] M. Blosi, S. Albonetti, F. Gatti, G. Baldi, and M. Dondi, "Au-Ag Nanoparticles as Red Pigment in Ceramic Inks for Digital Decoration," Dyes and Pigments, vol. 94, no. 2, pp. 355-362, 2012.

[43] P. Zijlstra, J. W. M. Chon, and M. Gu, "Five-Dimensional Optical Recording Mediated by Surface Plasmons in Gold Nanorods," Nature, vol. 459, no. 7245, pp. 410-413, 2009.

[44] X. Huang, P. K. Jain, I. H. El-Sayed, and M. A. El-Sayed, "Plasmonic Photothermal Therapy (Pptt) Using Gold Nanoparticles," Lasers in Medical Science, vol. 23, no. 3, p. 217, 2007.

[45] G. L. Liu, J. Kim, Y. Lu, and L. P. Lee, "Optofluidic Control Using Photothermal Nanoparticles," Nature Materials, vol. 5, no. 1, pp. 27-32, 2006.

[46] D. R. H. C. F. Bohren, "Extinction," in Absorption and Scattering of Light by Small Particles: Wiley interscience, 1983, pp. 286-324.

[47] X. Cui, F. Qin, Q. Ruan, X. Zhuo, and J. Wang, "Circular Gold Nanodisks with Synthetically Tunable Diameters and Thicknesses," Advanced Functional Materials, vol. 28, no. 11, p. 1705516, 2018.

[48] L. Scarabelli, "Recent Advances in the Rational Synthesis and Self-Assembly of Anisotropic Plasmonic Nanoparticles," in Pure and Applied Chemistry vol. 90, ed, 2018, p. 1393.

[49] F. Sohrabi and S. M. Hamidi, "Fabrication Methods of Plasmonic and Magnetoplasmonic Crystals: A Review," The European Physical Journal Plus, vol. 132, no. 1, p. 15, 2017.

[50] T. Sannomiya, O. Scholder, K. Jefimovs, C. Hafner, and A. B. Dahlin, "Investigation of Plasmon Resonances in Metal Films with Nanohole Arrays for Biosensing Applications," Small, vol. 7, no. 12, pp. 1653-1663, 2011.

[51] C. Langhammer, Z. Yuan, I. Zorić, and B. Kasemo, "Plasmonic Properties of Supported Pt and Pd Nanostructures," Nano Letters, vol. 6, no. 4, pp. 833-838, 2006.

[52] X. Cui, F. Qin, Q. Ruan, X. Zhuo, and J. Wang, "Circular Gold Nanodisks with Synthetically Tunable Diameters and Thicknesses," Advanced Functional Materials, vol. 28, no. 11, pp. 1-13, 2018.

[53] P. Hanarp, M. Käll, and D. S. Sutherland, "Optical Properties of Short Range Ordered Arrays of Nanometer Gold Disks Prepared by Colloidal Lithography," The Journal of Physical Chemistry B, vol. 107, no. 24, pp. 5768-5772, 2003.

[54] H. Xu and M. Käll, "Modeling the Optical Response of Nanoparticle-Based Surface Plasmon Resonance Sensors," Sensors and Actuators, B: Chemical, vol. 87, no. 2, pp. 244-249, 2002.

[55] T. Rindzevicius et al., "Nanohole Plasmons in Optically Thin Gold Films," The Journal of Physical Chemistry C, vol. 111, no. 3, pp. 1207-1212, 2007.

[56] E. S. H. Kang, H. Ekinge, and M. P. Jonsson, "Plasmonic Fanoholes: On the Gradual Transition from Suppressed to Enhanced Optical Transmission through Nanohole 
Arrays in Metal Films of Increasing Film Thickness," Optical Materials Express, vol. 9, no. 3, pp. 1404-1415, 2019.

[57] M. Quinten, A. Leitner, J. R. Krenn, and F. R. Aussenegg, "Electromagnetic Energy Transport Via Linear Chains of Silver Nanoparticles," Optics Letters, vol. 23, no. 17, pp. 1331-1331, 1998.

[58] B. Lamprecht et al., "Metal Nanoparticle Gratings: Influence of Dipolar Particle Interaction on the Plasmon Resonance," Physical Review Letters, vol. 84, no. 20, pp. 4721-4724, 2000.

[59] H. Fredriksson et al., "Hole-Mask Colloidal Lithography," Advanced Materials, vol. 19, no. 23, pp. 4297-4302, 2007.

[60] M. Cesaria et al., "Long- and Short-Range Ordered Gold Nanoholes as Large-Area Optical Transducers in Sensing Applications," Chemosensors, vol. 7, no. 1, pp. 13-13, 2019.

[61] B. Ai, Y. Yu, H. Möhwald, G. Zhang, and B. Yang, "Plasmonic Films Based on Colloidal Lithography," Advances in Colloid and Interface Science, vol. 206, pp. 5-16, 2014.

[62] J. Prikulis, P. Hanarp, L. Olofsson, D. Sutherland, and M. Käll, "Optical Spectroscopy of Nanometric Holes in Thin Gold Films," Nano Letters, vol. 4, no. 6, pp. 1003-1007, 2004.

[63] I. Zorić, B. Kasemo, C. Langhammer, and M. Zaaach, "Nanodisk Plasmons: Material Damping Mechanisms," ACS nano, vol. 5, no. 4, pp. 2535-2546, 2011.

[64] W.-S. Chang, B. Willingham, L. S. Slaughter, S. Dominguez-Medina, P. Swanglap, and S. Link, "Radiative and Nonradiative Properties of Single Plasmonic Nanoparticles and Their Assemblies," Accounts of Chemical Research, vol. 45, no. 11, pp. 1936-1945, 2012.

[65] P. Hernandez, P. J. Tandler, A. O. Govorov, M. T. Carlson, and H. H. Richardson, "Experimental and Theoretical Studies of Light-to-Heat Conversion and Collective Heating Effects in Metal Nanoparticle Solutions," Nano Letters, vol. 9, no. 3, pp. 11391146, 2009.

[66] G. Baffou, R. Quidant, and C. Girard, "Heat Generation in Plasmonic Nanostructures: Influence of Morphology," Applied Physics Letters, vol. 94, no. 15, pp. 1-3, 2009.

[67] E. S. H. Kang, M. S. Chaharsoughi, S. Rossi, and M. P. Jonsson, "Hybrid Plasmonic Metasurfaces," Journal of Applied Physics, vol. 126, no. 14, p. 140901, 2019.

[68] W. H. P. Pernice, "Finite-Difference Time-Domain Methods and Material Models for the Simulation of Metallic and Plasmonic Structures," Journal of Computational and Theoretical Nanoscience, vol. 7, no. 1, pp. 1-14, 2010.

[69] S. Burger, L. Zschiedrich, J. Pomplun, and F. Schmidt, "Finite Element Method for Accurate 3d Simulation of Plasmonic Waveguides," in Integrated Optics: Devices, Materials, and Technologie, 2010, vol. 7604: Proc SPIE.

[70] A. O. Govorov and H. H. Richardson, "Generating Heat with Metal Nanoparticles," Nano Today, vol. 2, no. 1, pp. 30-38, 2007.

[71] G. Palermo, T. Ritacco, D. Aceti, L. Pezzi, M. Giocondo, and A. De Luca, "PhotoThermal Effects in 1d Gratings of Gold Nanoparticles," Crystals, vol. 7, no. 1, pp. 1414, 2017.

[72] U. Guler et al., "Local Heating with Lithographically Fabricated Plasmonic Titanium Nitride Nanoparticles," Nano Letters, vol. 13, no. 12, p. 6078-6083, 2013.

[73] Y.-J. Yang and Y.-G. Lee, "Comparison of Plasmonic Structures in Terms of Temperature Increase under Equivalent Maximal Trapping Forces," Journal of Applied Physics, vol. 119, no. 8, p. 083108, 2016. 
[74] D. Tordera, D. Zhao, A. V. Volkov, X. Crispin, and M. P. Jonsson, "Thermoplasmonic Semitransparent Nanohole Electrodes," Nano Letters, vol. 17, no. 5, pp. 3145-3151, 2017.

[75] P. R. N. Childs, J. R. Greenwood, and C. A. Long, "Review of Temperature Measurement," Review of Scientific Instruments, vol. 71, no. 8, pp. 2959-2978, 2000.

[76] P. Sengupta and P. Garrity, "Sensing Temperature," Current biology : CB, vol. 23, no. 8, pp. R304-R307, 2013.

[77] D. Roncarati and V. Scarlato, "Regulation of Heat-Shock Genes in Bacteria: From Signal Sensing to Gene Expression Output," FEMS Microbiology Reviews, vol. 41, no. 4, pp. 549-574, 2017.

[78] C. R. McClung and S. J. Davis, "Ambient Thermometers in Plants: From Physiological Outputs Towards Mechanisms of Thermal Sensing," Current Biology, vol. 20, no. 24, pp. R1086-R1092, 2010.

[79] S. Eriksson, R. Hurme, and M. Rhen, "Low-Temperature Sensors in Bacteria," Philosophical transactions of the Royal Society of London. Series B, Biological sciences, vol. 357, no. 1423, pp. 887-893, 2002.

[80] K. L. Poff, "Temperature Sensing in Microorganisms Bt - Sensory Perception and Transduction in Aneural Organisms," Boston, MA, pp. 299-307: Springer US.

[81] E. O. Gracheva et al., "Molecular Basis of Infrared Detection by Snakes," Nature, vol. 464, no. 7291, pp. 1006-1011, 2010.

[82] Jennifer M. Enright et al., "Cyp27c1 Red-Shifts the Spectral Sensitivity of Photoreceptors by Converting Vitamin A1 into A2," Current Biology, vol. 25, no. 23, pp. 3048-3057, 2015.

[83] Q. Hua et al., "Skin-Inspired Highly Stretchable and Conformable Matrix Networks for Multifunctional Sensing," Nature Communications, vol. 9, no. 1, p. 244, 2018.

[84] J. Wade, T. Bhattacharjee, R. D. Williams, and C. C. Kemp, "A Force and Thermal Sensing Skin for Robots in Human Environments," Robotics and Autonomous Systems, vol. 96, pp. 1-14, 2017.

[85] A. M. Peier et al., "A Heat-Sensitive Trp Channel Expressed in Keratinocytes," Science, vol. 296, pp. 2046-2049, 2002.

[86] T. Lister, P. A. Wright, and P. H. Chappell, "Optical Properties of Human Skin," Journal of Biomedical Optics, vol. 17, no. 9, pp. 1-15, 2012.

[87] W. W. Lee et al., "A Neuro-Inspired Artificial Peripheral Nervous System for Scalable Electronic Skins," Science Robotics, vol. 4, no. 32, pp. eaax2198-eaax2198, 2019.

[88] S. Wang, J. Y. Oh, J. Xu, H. Tran, and Z. Bao, "Skin-Inspired Electronics: An Emerging Paradigm," Accounts of Chemical Research, vol. 51, no. 5, pp. 1033-1045, 2018.

[89] S. Zhao and R. Zhu, "Electronic Skin with Multifunction Sensors Based on Thermosensation," Advanced Materials, vol. 29, no. 15, p. 1606151, 2017.

[90] T. Q. Trung, S. Ramasundaram, B.-U. Hwang, and N.-E. Lee, "An All-Elastomeric Transparent and Stretchable Temperature Sensor for Body-Attachable Wearable Electronics," Advanced Materials, vol. 28, no. 3, pp. 502-509, 2016.

[91] K. Hu et al., "Self-Powered Electronic Skin with Biotactile Selectivity," Advanced Materials, vol. 28, no. 18, pp. 3549-3556, 2016.

[92] G. Y. Bae et al., "Pressure/Temperature Sensing Bimodal Electronic Skin with Stimulus Discriminability and Linear Sensitivity," Advanced Materials, vol. 30, no. 43, pp. 1803388-1803388, 2018.

[93] B. Maresca, C. Daraio, R. Di Giacomo, V. Costanza, and L. Bonanomi, "Biomimetic Temperature-Sensing Layer for Artificial Skins," Science Robotics, vol. 2, no. 3, pp. eaai9251-eaai9251, 2017. 
[94] T. Someya et al., "Conformable, Flexible, Large-Area Networks of Pressure and Thermal Sensors with Organic Transistor Active Matrixes," Proceedings of the National Academy of Sciences of the United States of America, vol. 102, pp. 1232112325, 2005.

[95] C. García Núñez, L. Manjakkal, and R. Dahiya, "Energy Autonomous Electronic Skin," npj Flexible Electronics, vol. 3, no. 1, pp. 1-1, 2019.

[96] Y. Gu et al., "Mini Review on Flexible and Wearable Electronics for Monitoring Human Health Information," Nanoscale Research Letters, vol. 14, no. 1, pp. 263-263, 2019.

[97] R. A. Kishore et al., "Energy Scavenging from Ultra-Low Temperature Gradients," Energy \& Environmental Science, vol. 12, no. 3, pp. 1008-1018, 2019.

[98] D. Zabek and F. Morini, "Solid State Generators and Energy Harvesters for Waste Heat Recovery and Thermal Energy Harvesting," Thermal Science and Engineering Progress, vol. 9, pp. 235-247, 2019.

[99] H. Tong et al., "Harvest Green Energy through Energy Recovery from Waste: A Technology Review and an Assessment of Singapore," Renewable and Sustainable Energy Reviews, vol. 98, pp. 163-178, 2018.

[100] T. Ding et al., "Hybrid Photothermal Pyroelectric and Thermogalvanic Generator for Multisituation Low Grade Heat Harvesting," Advanced Energy Materials, vol. 8, no. 33, p. 1802397, 2018.

[101] O. H. Ando Junior, A. L. O. Maran, and N. C. Henao, "A Review of the Development and Applications of Thermoelectric Microgenerators for Energy Harvesting," Renewable and Sustainable Energy Reviews, vol. 91, pp. 376-393, 2018.

[102] S. M. Pourkiaei et al., "Thermoelectric Cooler and Thermoelectric Generator Devices: A Review of Present and Potential Applications, Modeling and Materials," Energy, vol. 186, p. 115849, 2019.

[103] F. J. DiSalvo, "Thermoelectric Cooling and Power Generation," Science, vol. 285, no. 5428, pp. 703-706, 1999.

[104] D. S. Patil, R. R. Arakerimath, and P. V. Walke, "Thermoelectric Materials and Heat Exchangers for Power Generation - a Review," Renewable and Sustainable Energy Reviews, vol. 95, pp. 1-22, 2018.

[105] S. Sharma, V. K. Dwivedi, S. N. Pandit, S. Sharma, V. K. Dwivedi, and S. N. Pandit, "A Review of Thermoelectric Devices for Cooling Applications," vol. 5075, 2014.

[106] B. Xu and M. J. Verstraete, "First Principles Explanation of the Positive Seebeck Coefficient of Lithium," Physical Review Letters, vol. 112, no. 19, p. 196603, 2014.

[107] D. Ponnamma, G. J. Ogunleye, P. Sharma, and M. A. AlMaadeed, "Piezo- and Thermoelectric Materials from Biopolymer Composites," in Biopolymer Composites in Electronics, K. K. Sadasivuni, D. Ponnamma, J. Kim, J. J. Cabibihan, and M. A. AlMaadeed, Eds.: Elsevier, 2017, pp. 333-352.

[108] Y. Mishima, Y. Kimura, and S. Wng Kim, "Enhancement of Thermoelectric Figure of Merit through Nanostructural Control on Intermetallic Semiconductors toward HighTemperature Applications," in Nanomaterials, H. Hosono et al., Eds. Oxford: Elsevier Science Ltd, 2006, pp. 383-418.

[109] H. J. Goldsmid, "Thermoelectric Properties of Metals and Semiconductors," in Introduction to Thermoelectricity, vol. 121: Springer, 2016, pp. 25-44.

[110] D. Beretta et al., "Thermoelectrics: From History, a Window to the Future," Materials Science and Engineering: R: Reports, vol. 138, no. 12, 2018.

[111] S. Duhr and D. Braun, "Why Molecules Move Along a Temperature Gradient," Proceedings of the National Academy of Sciences, vol. 103, no. 52, pp. 19678-19682, 2006. 
[112] T. Soret, "Is Soret Equilibrium a Non-Equilibrium Effect?," Comptes Rendus Mécanique, vol. 341, no. 4, pp. 438-448, 2013.

[113] E. L. Talbot, J. Kotar, L. Parolini, L. Di Michele, and P. Cicuta, "Thermophoretic Migration of Vesicles Depends on Mean Temperature and Head Group Chemistry," Nature communications, vol. 8, pp. 15351-15351, 2017.

[114] A. Parola and R. Piazza, "Particle Thermophoresis in Liquids," The European Physical Journal E, vol. 15, no. 3, pp. 255-263, 2004.

[115] P. Costesèque, A. Mojtabi, and J. K. Platten, "Thermodiffusion Phenomena," Comptes Rendus Mécanique, vol. 339, no. 5, pp. 275-279, 2011.

[116] D. Zhao et al., "Polymer Gels with Tunable Ionic Seebeck Coefficient for UltraSensitive Printed Thermopiles," Nature Communications, vol. 10, no. 1, p. 1093, 2019.

[117] I. Petsagkourakis, K. Tybrandt, X. Crispin, I. Ohkubo, and T. Mori, "Thermoelectric Materials and Applications for Energy Harvesting Power Generation," Science and Technology of Advanced Materials, vol. 19, no. 1, pp. 836-862, 2018.

[118] M. Bonetti, S. Nakamae, M. Roger, and P. Guenoun, "Huge Seebeck Coefficients in Nonaqueous Electrolytes," The Journal of Chemical Physics, vol. 134, no. 11, p. $114513,2011$.

[119] H. Wang et al., "Ionic Thermoelectric Figure of Merit for Charging of Supercapacitors," Advanced Electronic Materials, vol. 3, no. 4, p. 1700013, 2017.

[120] F. Jiao et al., "Correction: Ionic Thermoelectric Paper," Journal of Materials Chemistry $A$, vol. 5, no. 37, pp. 20053-20053, 2017.

[121] T. J. Abraham, D. R. MacFarlane, and J. M. Pringle, "High Seebeck Coefficient Redox Ionic Liquid Electrolytes for Thermal Energy Harvesting," Energy \& Environmental Science, vol. 6, no. 9, pp. 2639-2645, 2013.

[122] K. H. Lee, M. S. Kang, S. Zhang, Y. Gu, T. P. Lodge, and C. D. Frisbie, "'Cut and Stick" Rubbery Ion Gels as High Capacitance Gate Dielectrics," Advanced Materials, vol. 24, no. 32, pp. 4457-4462, 2012.

[123] M. Bonetti, S. Nakamae, B. T. Huang, T. J. Salez, C. Wiertel-Gasquet, and M. Roger, "Thermoelectric Energy Recovery at Ionic-Liquid/Electrode Interface," The Journal of Chemical Physics, vol. 142, no. 24, p. 244708, 2015.

[124] T. Welton, "Ionic Liquids: A Brief History," Biophysical Reviews, vol. 10, no. 3, pp. 691-706, 2018.

[125] A. K. Pathak, C. Ameta, R. Ameta, and P. B. Punjabi, "Microwave-Assisted Organic Synthesis in Ionic Liquids," Journal of Heterocyclic Chemistry, vol. 53, no. 6, pp. 1697 $1705,2016$.

[126] Z. Lei, B. Chen, Y.-M. Koo, and D. R. MacFarlane, "Introduction: Ionic Liquids," Chemical Reviews, vol. 117, no. 10, pp. 6633-6635, 2017.

[127] J. S. Wilkes and M. J. Zaworotko, "Air and Water Stable 1-Ethyl-3-Methylimidazolium Based Ionic Liquids," Journal of the Chemical Society, Chemical Communications, no. 13, pp. 965-967, 1992.

[128] E. Laux, S. Uhl, T. Journot, J. Brossard, L. Jeandupeux, and H. Keppner, "Aspects of Protonic Ionic Liquid as Electrolyte in Thermoelectric Generators," Journal of Electronic Materials, vol. 45, no. 7, pp. 3383-3389, 2016.

[129] S. W. H. Hasan, S. M. Said, A. S. Abu Bakar, H. A. Jaffery, M. Faizul, and M. Sabri, "The Role of Electrolyte Fluidity on the Power Generation Characteristics of Thermally Driven Electrochemical Cells," Sains Malaysiana, vol. 47, no. 2, pp. 403-408, 2018.

[130] A. P. Fröba, M. H. Rausch, K. Krzeminski, D. Assenbaum, P. Wasserscheid, and A. Leipertz, "Thermal Conductivity of Ionic Liquids: Measurement and Prediction," International Journal of Thermophysics, vol. 31, no. 11, pp. 2059-2077, 2010. 
[131] L. Rebenklau et al., "Temperature Sensors Based on Thermoelectric Effect," in 2015 European Microelectronics Packaging Conference (EMPC), 2015, pp. 1-5.

[132] M. Shiran Chaharsoughi, D. Zhao, X. Crispin, S. Fabiano, and M. P. Jonsson, "Thermodiffusion-Assisted Pyroelectrics - Enabling Rapid and Stable Heat and Radiation Sensing," Advanced Functional Materials, vol. 29, no. 28, pp. 1900572$1900581,2019$.

[133] D. Zhao et al., "Ionic Thermoelectric Supercapacitors," Energy \& Environmental Science, vol. 9, no. 4, pp. 1450-1457, 2016.

[134] A. Cuadras, M. Gasulla, and V. Ferrari, "Thermal Energy Harvesting through Pyroelectricity," Sensors and Actuators A: Physical, vol. 158, no. 1, pp. 132-139, 2010.

[135] R. W. Whatmore, "Pyroelectric Devices and Materials," Reports on Progress in Physics, vol. 49, no. 12, p. 1335, 1986.

[136] R. W. Whatmore and R. Watton, "Pyroelectric Materials and Devices," in Infrared Detectors and Emitters: Materials and Devices, P. Capper and C. T. Elliott, Eds. Boston, MA: Springer US, 2001, pp. 99-147.

[137] M. H. Lee, R. Guo, and A. S. Bhalla, "Pyroelectric Sensors," Journal of Electroceramics, vol. 2, no. 4, pp. 229-242, 1998.

[138] A. Agulyansky, "Ferroelectric Properties of Niobium and Tantalum Fluoride Compounds," in Chemistry of Tantalum and Niobium Fluoride Compounds, A. Agulyansky, Ed. Amsterdam: Elsevier Science, 2004, pp. 217-220.

[139] V. Ming Hong Ng, L. Bing Kong, W. Que, C. Wang, S. Li, and T. Zhang, "Pyroelectric Materials," in Comprehensive Energy Systems, vol. 2, I. Dincer, Ed. Oxford: Elsevier, 2018, pp. 740-759.

[140] K. C. Kao, "Electric Polarization and Relaxation," in Dielectric Phenomena in Solids, K. C. Kao, Ed. San Diego: Academic Press, 2004, pp. 41-114.

[141] R. Dorey, "Microstructure-Property Relationships: How the Microstructure of the Film Affects Its Properties," in Ceramic Thick Films for Mems and Microdevices, R. Dorey, Ed. Oxford: William Andrew Publishing, 2012, pp. 85-112.

[142] K. C. Kao, "Ferroelectrics, Piezoelectrics, and Pyroelectrics," in Dielectric Phenomena in Solids, vol. 4, K. C. Kao, Ed. San Diego: Academic Press, 2004, pp. 213-282.

[143] S. B. Lang and S. Muensit, "Review of Some Lesser-Known Applications of Piezoelectric and Pyroelectric Polymers," Applied Physics A, vol. 85, no. 2, pp. 125134, 2006.

[144] D. Mao, B. E, and M. A, "Ferroelectric Properties and Polarization Switching Kinetic of Poly (Vinylidene Fluoride-Trifluoroethylene) Copolymer," Ferroelectrics - Physical Effects, 2011.

[145] S. Gael, G. Daniel, and A. Amen, "On Thermoelectric and Pyroelectric Energy Harvesting," Smart Materials and Structures, vol. 18, no. 12, p. 125006, 2009.

[146] A. V. Bune et al., "Piezoelectric and Pyroelectric Properties of Ferroelectric LangmuirBlodgett Polymer Films," Journal of Applied Physics, vol. 85, no. 11, pp. 7869-7873, 1999.

[147] M. Poulsen and S. Ducharme, "Why Ferroelectric Polyvinylidene Fluoride Is Special," vol. 17, no. 4, pp. 1028 - 1035, 2010.

[148] M. Mai, S. Ke, P. Lin, and X. Zeng, "Ferroelectric Polymer Thin Films for Organic Electronics," Journal of Nanomaterials, vol. 2015, pp. 1-14, 2015.

[149] M. Shiran Chaharsoughi et al., "Hybrid Plasmonic and Pyroelectric Harvesting of Light Fluctuations," Advanced Optical Materials, vol. 6, no. 11, p. 1701051, 2018.

[150] S. R. Hoh, "Conversion of Thermal to Electrical Energy with Ferroelectric Materials," Proceedings of the IEEE, vol. 51, no. 5, pp. 838-845, 1963. 
[151] D. Guyomar, G. Sebald, E. Lefeuvre, and A. Khodayari, "Toward Heat Energy Harvesting Using Pyroelectric Material," Journal of Intelligent Material Systems and Structures, vol. 20, no. 3, pp. 265-271, 2009.

[152] C. R. Bowen, J. Taylor, E. LeBoulbar, D. Zabek, A. Chauhan, and R. Vaish, "Pyroelectric Materials and Devices for Energy Harvesting Applications," Energy \& Environmental Science, vol. 7, no. 12, pp. 3836-3856, 2014.

[153] Z. Wang et al., "Light-Induced Pyroelectric Effect as an Effective Approach for Ultrafast Ultraviolet Nanosensing," Nature Communications, vol. 6, pp. 1-7, 2015.

[154] A. Al-zubaidi, X. Ji, and J. Yu, "Thermal Charging of Supercapacitors: A Perspective," Sustainable Energy \& Fuels, vol. 1, no. 7, pp. 1457-1474, 2017.

[155] X. Shi, S. Zheng, Z.-S. Wu, and X. Bao, "Recent Advances of Graphene-Based Materials for High-Performance and New-Concept Supercapacitors," Journal of Energy Chemistry, vol. 27, no. 1, pp. 25-42, 2018.

[156] R. Brooke et al., "Supercapacitors on Demand: All-Printed Energy Storage Devices with Adaptable Design," Flexible and Printed Electronics, vol. 4, no. 1, pp. 1500615006, 2019.

[157] P. K. Das, "Effect of Temperature on Zeta Potential of Functionalized Gold Nanorod," Microfluidics and Nanofluidics, vol. 21, no. 5, p. 95, 2017.

[158] H. Lim, W. Lu, and Y. Qiao, "Dependence on Cation Size of Thermally Induced Capacitive Effect of a Nanoporous Carbon," Applied Physics Letters, vol. 101, no. 6, p. 063902, 2012.

[159] P. Khadkikar, "The Principles and Properties of Thermostat Metals," JOM, vol. 45, no. 6, pp. 39-42, 1993.

[160] S. B. Reddy. (October 2019). Thermostat Working Principle. Available: https://instrumentationtools.com/thermostat-working-principle/

[161] K. A. I. Siren, G. RosÉN, J. Vad, and P. V. Nielsen, "Experimental Techniques," in Industrial Ventilation Design Guidebook, H. Goodfellow and E. Tähti, Eds. San Diego: Academic Press, 2001, pp. 1105-1195.

[162] A. S. Morris and R. Langari, "Temperature Measurement," in Measurement and Instrumentation, A. S. Morris and R. Langari, Eds. Boston: Butterworth-Heinemann, 2012, pp. 347-396.

[163] J. Fraden, "Data Acquisition," in Handbook of Modern Sensors: Physics, Designs, and Applications New York, NY: Springer New York, 2010, pp. 1-12.

[164] A. Ostadfar, "Real Time Measurement Techniques of Biofluids," in Biofluid Mechanics, A. Ostadfar, Ed.: Academic Press, 2016, pp. 295-322.

[165] S. Basu and A. K. Debnath, "General Instruments," in Power Plant Instrumentation and Control Handbook, S. Basu and A. K. Debnath, Eds. Boston: Academic Press, 2015, pp. 229-296.

[166] I. Poole. (October 2019). Thermistor. Available: https://www.electronicsnotes.com/articles/electronic_components/resistors/thermistor-technology-types.php

[167] N. B. Stevens, "Radiation Thermopiles," in Semiconductors and Semimetals, vol. 5, R. K. Willardson and A. C. Beer, Eds.: Elsevier, 1970, pp. 287-318.

[168] F. A. Firestone, "Radiation Thermopile Design," Review of Scientific Instruments, vol. 1, no. 11, pp. 630-649, 1930.

[169] Basic Electronics Tutorials. Teperature Sensors. Available: https://www.electronicstutorials.ws/io/io 3.html

[170] P. L. Richards, "Bolometers for Infrared and Millimeter Waves," Journal of Applied Physics, vol. 76, no. 1, pp. 1-24, 1994.

[171] F. Zhang, Y. Zang, D. Huang, C. A. Di, and D. Zhu, "Flexible and Self-Powered Temperature-Pressure Dual-Parameter Sensors Using Microstructure-Frame-Supported 
Organic Thermoelectric Materials," Nature Communications, vol. 6, no. May, pp. 1-10, 2015.

[172] J. Rao, Z. Chen, D. Zhao, Y. Yin, X. Wang, and F. Yi, "Recent Progress in Self-Powered Skin Sensors," Sensors, vol. 19, no. 12, 2019.

[173] S. Chun, W. Son, H. Kim, S. K. Lim, C. Pang, and C. Choi, "Self-Powered Pressureand Vibration-Sensitive Tactile Sensors for Learning Technique-Based Neural Finger Skin," Nano Letters, vol. 19, no. 5, pp. 3305-3312, 2019.

[174] J. Chun et al., "Self-Powered Temperature-Mapping Sensors Based on ThermoMagneto-Electric Generator," ACS Applied Materials \& Interfaces, vol. 10, no. 13, pp. 10796-10803, 2018.

[175] W. Han et al., "A Self-Powered Wearable Noninvasive Electronic-Skin for Perspiration Analysis Based on Piezo-Biosensing Unit Matrix of Enzyme/Zno Nanoarrays," ACS Applied Materials \& Interfaces, vol. 9, no. 35, pp. 29526-29537, 2017.

[176] X. Pu, W. Hu, and Z. L. Wang, "Toward Wearable Self-Charging Power Systems: The Integration of Energy-Harvesting and Storage Devices," Small, vol. 14, no. 1, pp. 1-19, 2018.

[177] R. G. Larson and T. J. Rehg, "Spin Coating," in Liquid Film Coating: Scientific Principles and Their Technological Implications, S. F. Kistler and P. M. Schweizer, Eds. Dordrecht: Springer Netherlands, 1997, pp. 709-734.

[178] H. Adachi and K. Wasa, "Thin Films and Nanomaterials," in Handbook of Sputtering Technology (Second Edition), K. Wasa, I. Kanno, and H. Kotera, Eds. Oxford: William Andrew Publishing, 2012, pp. 3-39.

[179] H. Fredriksson et al., "Hole-Mask Colloidal Lithography," Advanced Materials, vol. 19, no. 23, pp. 4297-4302, 2007.

[180] M. Cesaria, A. Taurino, M. G. Manera, M. Minunni, S. Scarano, and R. Rella, "Gold Nanoholes Fabricated by Colloidal Lithography: Novel Insights into Nanofabrication, Short-Range Correlation and Optical Properties," Nanoscale, vol. 11, no. 17, pp. 84168432, 2019.

[181] A. Marcano Olaizola, "Photothermal Determination of Absorption and Scattering Spectra of Silver Nanoparticles," Applied Spectroscopy, vol. 72, no. 2, pp. 234-240, 2018.

[182] Y. Nishijima, L. Rosa, and S. Juodkazis, "Surface Plasmon Resonances in Periodic and Random Patterns of Gold Nano-Disks for Broadband Light Harvesting," Optics Express, vol. 20, no. 10, pp. 11466-11477, 2012.

[183] S. A. Choudhury and M. H. Chowdhury, "Use of Plasmonic Metal Nanoparticles to Increase the Light Absorption Efficiency of Thin-Film Solar Cells," in IEEE International Conference on Sustainable Energy Technologies (ICSET), Hanoi, 2016, pp. 196-201.

[184] M. C. Beard, J. M. Luther, and A. J. Nozik, "The Promise and Challenge of Nanostructured Solar Cells," Nature Nanotechnology, vol. 9, no. 12, pp. 951-954, 2014.

[185] C. H. Liow et al., "Spatially Probed Plasmonic Photothermic Nanoheater Enhanced Hybrid Polymeric-Metallic Pvdf-Ag Nanogenerator," Small, vol. 14, no. 7, p. 1702268, 2018.

[186] K. Xu, Y. Lu, and K. Takei, "Multifunctional Skin-Inspired Flexible Sensor Systems for Wearable Electronics," Advanced Materials Technologies, vol. 4, no. 3, p. 1800628, 2019.

[187] J. W. Stewart, J. H. Vella, W. Li, S. Fan, and M. H. Mikkelsen, "Ultrafast Pyroelectric Photodetection with on-Chip Spectral Filters," Nature Materials, 2019.

[188] "Skin to E-Skin," Nature Nanotechnology, vol. 12, no. 11, pp. 1017-1017, 2017. 
[189] A. Ahiabu and M. J. Serpe, "Rapidly Responding Ph- and Temperature-Responsive Poly (N-Isopropylacrylamide)-Based Microgels and Assemblies," ACS Omega, vol. 2, no. 5, pp. 1769-1777, 2017.

[190] N. Sharma et al., "Tunable Plasmonic Nanohole Arrays Actuated by a Thermoresponsive Hydrogel Cushion," The Journal of Physical Chemistry C, vol. 120, no. 1, pp. 561-568, 2016. 


\section{Part II}

Papers 


\section{Papers}

The papers associated with this thesis have been removed for copyright reasons. For more details about these see:

http://urn.kb.se/resolve?urn=urn:nbn:se:liu:diva-163318 


\section{FACULTY OF SCIENCE AND ENGINEERING}

Linköping Studies in Science and Technology, Dissertation No. 2045, 2020 Department of Science and Technology

Linköping University

SE-581 83 Linköping, Sweden

www.liu.se

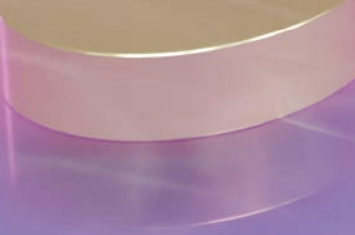

